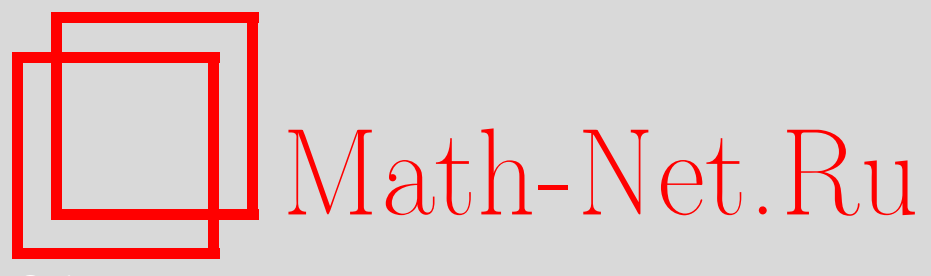

А. Г. Басуев, Гамильтониан границы раздела фаз и фазовые переходы первого рода. Обобщение теоремы ЛиЯнга, ТМФ, 2007, том 153, номер 1, 98-123

DOI: https://doi.org/10.4213/tmf6124

Использование Общероссийского математического портала Math-Net.Ru подразумевает, что вы прочитали и согласны с пользовательским соглашением http://www.mathnet.ru/rus/agreement

Параметры загрузки:

IP : 54.172 .240 .79

26 апреля 2023 г., 13:01:09

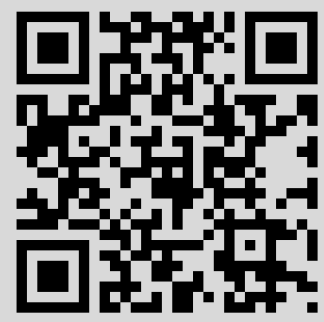




\title{
ГАМИЛЬТОНИАН ГРАНИЦЫ РАЗДЕЛА ФАЗ И ФАЗОВЫЕ ПЕРЕХОДЫ ПЕРВОГО РОДА. ОБОБЩЕНИЕ ТЕОРЕМЫ ЛИ-ЯНГА
}

\begin{abstract}
Проведено обобщение теории Пирогова-Синая, доказаны результаты, применимые при рассмотрении фазовых переходов первого рода как в случае объемных, так и в случае поверхностных фаз решетчатых моделей. Область фазовых переходов первого рода расширяется по активностям до всего комплексного пространства $\mathbb{C}^{\Phi}$, где $\Phi$ - множество фаз модели. Доказано обобщение теоремы Ли-Янга: статистические суммы с устойчивым граничным условием как функции активностей не имеют нулей в $\mathbb{C}^{\Phi}$.
\end{abstract}

Ключевые слова: теория Пирогова-Синая, многофазная контурная модель, гамильтониан границы, кластерное разложение гамильтониана границы, контурные уравнения, уравнение состояния, фазовая диаграмма, fс-инвариантность многофазных контурных моделей.

\section{1. ВВЕДЕНИЕ}

Круг работ, в которых построены обобщения теории Пирогова-Синая [1] и при помощи этой теории и ее обобщений получены значимые результаты, достаточно велик, см., например, работы [2]-[14] и [15]-[18] $]^{1)}$. Результаты работы [9] и данной работы, являющейся продолжением [9], применимы к решетчатым моделям с счетным числом фаз, с нефинитным многочастичным взаимодействием, убывающим быстрее некоторой степени, при произвольных комплексных внешних полях. В области фазовых переходов первого рода построены сходящиеся разложения термодинамических и корреляционных функций. Кроме того, в настоящей работе доказана теорема I.4 ${ }^{2}$ (доказательство в случае нефинитного взаимодействия не было опубликовано ранее) и ее обобщение - теорема 1, на которой будет основано изучение поверхностных фаз. Область фазовых переходов первого рода расширена по активностям до $\mathbb{C}^{\Phi}$, где $\Phi$ - множество фаз. Последнее утверждение можно сформулировать следующим образом: статистические суммы с устойчивым граничным

\footnotetext{
1) Более полный список работ, опубликованных к началу 90-х гг, см. в [16].

2) Здесь и далее ссылки на работы [9] даются в следующем виде: теорема I.4 означает теорему 4 первой работы из [9], формула (II.30) - это формула (30) второй из работ [9] и т.п.
}

* Санкт-Петербургский государственный университет технологии и дизайна, Санкт-Петербург, Россия. E-mail: Basuev@math.sutd.ru 
условием не имеют нулей в области $\operatorname{Re} p_{q_{1}}<\max \operatorname{Re} p_{q}$, где $p_{q}$ - давление фазы $q$. Ранее было известно [5], [9], что эта область содержит $\mathbb{R}^{\Phi}$, а также комплексную окрестность каждого страта фазовой диаграммы, сам страт представляет собой комплексное многообразие (см. также [15]). В работе Исакова [19] доказано, что свободная энергия в модели Изинга имеет существенную особенность на единичной окружности в плоскости активностей (см. также [20]). В дальнейшем Фридли и Пфистером в работе [21] результат Исакова был обобщен на двухфазные контурные модели. Можно предполагать, что в многофазном случае в условиях теоремы 4 точки границы области аналитичности являются одновременно точками существенных особенностей давления. В работе [18] исследуются нули статистических сумм с периодическими граничными условиями при помощи теории Пирогова-Синая. Результаты настоящей работы касаются статистических сумм с постоянными граничными условиями ${ }^{3)}$.

Основные результаты настоящей работы формулируются в теоремах 1 и 4 (разделы 2 и 3). Основной шаг доказательства теоремы 1 сформулирован в виде теоремы 3. В работе доказан критерий устойчивости фаз (теорема 2), полезный при анализе фазовых диаграмм. В разделе 4 область фазовых переходов первого рода расширяется по внешним полям до $\mathbb{C}^{\Phi}$. Доказательство этого факта базируется на теореме 1 , а также на тождестве (52) для статистических сумм многофазной контурной модели. Конечно, это не значит, что давление аналитично в $\mathbb{C}^{\Phi}$. Интересно отметить, что мнимая часть давления, найденного по устойчивому граничному условию, зависит от этого условия, а вещественная часть от него не зависит, в отличие от вещественного случая, где доказана независимость давления от граничного условия [22]-[24]. Равенство мнимых частей давлений для устойчивых граничных условий сужает $C^{\infty}$-гладкий страт сосуществования фаз до комплексного многообразия или аналитического множества. Можно полагать, что полученные результаты и, в частности, тождество (52) делают более прозрачной гипотезу Ли-Янга [25] о фазовых переходах, а также улучшают понимание аналитических свойств моделей, по крайней мере, в области фазовых переходов первого рода (см. обсуждение Рюэлем этого вопроса в разделе 5.7 работы [23]).

\section{2. ОПРЕДЕЛЕНИЯ, ОСНОВНАЯ ТЕОРЕМА И КРИТЕРИЙ УСТОЙЧИВОСТИ ФАЗ}

Мы рассматриваем в основном решетчатые модели в $\mathbb{Z}^{\nu}, \nu \geqslant 2$, хотя ясно, что необходимая модификация построений может быть применена также к ряду ситуаций, рассмотренных в других работах, использующих теорию Пирогова-Синая. Нам удобно считать, что многие геометрические объекты, например, поверхность, отделяющая разные спины данной конфигурации, естественно вложены в $\mathbb{R}^{\nu}$. Приведем определение многофазной контурной модели ("усеченное" определение раздела I.2).

ОПРЕДЕЛЕНИЕ 1. Будем говорить, что определена многофазная контурная модель, если задано множество "названий фаз" $\Phi$, химические потенциалы (или внешние поля) $h_{q}, q \in \Phi$, множество границ $\gamma$, гамильтониан границы $H(\vec{\partial}), \vec{\partial} \in \gamma$. Кроме того, справедливо следующее.

\footnotetext{
3) В работе [18] содержится достаточно полная библиография по обобщению теоремы Ли-Янга.
} 
1. Каждой границе $\vec{\partial} \in \gamma$ сопоставлено "векторное разбиение" $\mathbb{R}^{\nu}$ на носитель границы $V_{\vec{\partial}}$ и связные компоненты $\mathbb{R}^{\nu} \backslash V_{\vec{\partial}}$, каждая из которых "занята" ровно одной фазой $k \in \Phi$. Из всех компонент $\mathbb{R}^{\nu} \backslash V_{\vec{\partial}}$ лишь одна компонента бесконечна, обозначим ее $V_{\text {ех }}(\overrightarrow{\boldsymbol{\partial}})$, положив $V(\overrightarrow{\boldsymbol{\partial}}):=\mathbb{R}^{\nu} \backslash V_{\mathrm{ex}}(\overrightarrow{\boldsymbol{\partial}})^{4)}$, фазу бесконечной компоненты обозначим $k_{\text {ex }}(\vec{\partial})$. Множество номеров конечных компонент $\mathbb{R}^{\nu} \backslash V_{\vec{\partial}}$ будем обозначать как $l(\overrightarrow{\boldsymbol{\partial}})$, соответствующие фазы как $k_{i}(\overrightarrow{\boldsymbol{\partial}}), i \in l(\overrightarrow{\boldsymbol{\partial}})$, объединение всех компонент с данным $q$ будем обозначать $V_{q}(\overrightarrow{\boldsymbol{\partial}})$, множество номеров всех компонент $\mathbb{R}^{\nu} \backslash V_{\overrightarrow{\boldsymbol{\partial}}}$ будем обозначать $\bar{l}(\vec{\partial})$. Среди границ есть границы с пустым носителем, для каждой из которых $\mathbb{R}^{\nu}$ "занято" фазой $q$. Множество всех границ, у которых бесконечная компонента занята фазой $q$, а носитель лежит в $V$, обозначим $\gamma_{q}(V), \gamma_{q}:=\gamma_{q}\left(\mathbb{Z}^{\nu}\right)$.

2. Для каждой границы задано разбиение на "простейшие границы", назваемые контурами, $\vec{\partial}:=\bigcup_{\vec{\Gamma} \in \vec{\partial}} \vec{\Gamma}, V_{\vec{\partial}}:=\bigcup_{\vec{\Gamma} \in \vec{\partial}} V_{\vec{\Gamma}}$ так, что типы фаз, примыкающих к носителю контура, те же, что и в границе. Носители разных контуров границы не пересекаются. Множество контуров границы, для каждого из которых носитель $V_{\overrightarrow{\boldsymbol{\partial}} \backslash \overrightarrow{\boldsymbol{\Gamma}}}$ содержится в некоторой компоненте $V_{i}(\overrightarrow{\boldsymbol{\Gamma}})$, обозначим $\min ^{0} \overrightarrow{\boldsymbol{\partial}}$. Множеству контуров $\overrightarrow{\boldsymbol{\partial}} \backslash \overrightarrow{\boldsymbol{\Gamma}}, \overrightarrow{\boldsymbol{\Gamma}} \in \min ^{0} \overrightarrow{\boldsymbol{\partial}}$, также соответствует ровно одна граница с очевидным образом определенными носителем и соответствующим векторным разбиением $\mathbb{R}^{\nu}$. Все границы, состоящие из контуров границы $\overrightarrow{\boldsymbol{\partial}}$, обозначим как $\gamma(\overrightarrow{\boldsymbol{\partial}})$. Ясно, что $\min ^{0} \vec{\partial}:=\{\overrightarrow{\boldsymbol{\Gamma}} \in \vec{\partial}: \vec{\partial} \backslash \overrightarrow{\boldsymbol{\Gamma}} \in \gamma(\vec{\partial})\}$

3. Статистическая сумма многофазной контурной модели в объеме $V$ с граничным условием $q$ задается следующим образом $\left(z_{q}:=e^{h_{q}}\right)$ :

$$
\Xi_{q}(V, H):=\sum_{\overrightarrow{\boldsymbol{\partial}} \in \gamma_{q}(V)} z_{q}^{V \backslash V(\overrightarrow{\boldsymbol{\partial}})} e^{-H(\overrightarrow{\boldsymbol{\partial}})} \prod_{i \in l(\overrightarrow{\boldsymbol{\partial}})} z_{k_{i}(\overrightarrow{\boldsymbol{\partial}})}^{V_{i}(\overrightarrow{\boldsymbol{\partial}})} ;
$$

для конкретных моделей будем приводить статистическую сумму к виду $(V \subset \Lambda)$

$$
\Xi_{q}^{\Lambda}(V, H):=\sum_{\overrightarrow{\boldsymbol{\partial}} \in \gamma_{q}(V)} e^{-H_{f}^{\Lambda}(\overrightarrow{\boldsymbol{\partial}})},
$$

величину $H_{f}^{\Lambda}(\overrightarrow{\boldsymbol{\partial}})$ назовем полным гамильтонианом границы.

ОПРЕДЕЛЕНИЕ 2. Будем говорить, что для многофазной контурной модели задано кластерное разложение гамильтониана границы, если определены функционалы $H_{0}^{\Lambda}(\overrightarrow{\boldsymbol{\Gamma}})$, а также "многочастичные потенциалы" взаимодействия контуров $W^{\Lambda}(V \mid \overrightarrow{\boldsymbol{\partial}})$ такие, что

$$
H_{f}^{\Lambda}(\overrightarrow{\boldsymbol{\partial}}):=\sum_{\overrightarrow{\boldsymbol{\Gamma}} \in \overrightarrow{\boldsymbol{\partial}}} H_{0}^{\Lambda}(\overrightarrow{\boldsymbol{\Gamma}})+\sum_{V: V \cap \Lambda \neq \varnothing} W^{\Lambda}(V \mid \overrightarrow{\boldsymbol{\partial}}) .
$$

В дальнейшем $H_{0}^{\Lambda}(\overrightarrow{\boldsymbol{\partial}}):=\sum_{\overrightarrow{\boldsymbol{\Gamma}} \in \overrightarrow{\boldsymbol{\partial}}} H_{0}^{\Lambda}(\overrightarrow{\boldsymbol{\Gamma}})$. Введем величины

$$
h_{q}^{\Lambda}(x):=-\sum_{V \ni x} \frac{W^{\Lambda}(V \mid q)}{|V \cap \Lambda|} .
$$

\footnotetext{
4) В работе будем использовать обозначения $A:=B$ или $B=: A$, когда $A$ равно $B$ по определению.
} 
Пусть $W^{\Lambda}(V \mid q)=W(V \mid q)$ при $V \subset \Lambda$ и потенциалы $W(V \mid q)$ трансляционно-инвариантны. Тогда $h_{q}^{\Lambda}(x) \underset{\Lambda \uparrow Z^{\nu}}{\longrightarrow} h_{q}$, где

$$
h_{q}:=-\sum_{V \ni 0} \frac{W(V \mid q)}{|V|}
$$

Из (3)-(5) нетрудно получить, что

$$
H_{f}^{\Lambda}(\overrightarrow{\boldsymbol{\partial}})=H^{\Lambda}(\overrightarrow{\boldsymbol{\partial}})+\sum_{x \in \Lambda} h_{q}^{\Lambda}(x)-h_{q}|V(\overrightarrow{\boldsymbol{\partial}})|+\sum_{i \in l(\overrightarrow{\boldsymbol{\partial}})} h_{k_{i}}\left|V_{i}(\overrightarrow{\boldsymbol{\partial}})\right|+c_{q}(\Lambda),
$$

где $q$ - внешняя фаза границы $\vec{\partial}$,

$$
\begin{aligned}
H^{\Lambda}(\overrightarrow{\boldsymbol{\partial}}):=H_{0}^{\Lambda}(\overrightarrow{\boldsymbol{\partial}})+ & \sum_{V: V \cap \Lambda \neq \varnothing}\left[W^{\Lambda}(V \mid \overrightarrow{\boldsymbol{\partial}})-\sum_{q_{1}} \frac{\left|V_{q_{1}}(\overrightarrow{\boldsymbol{\partial}}) \cap V \cap \Lambda\right|}{|V|} W\left(V \mid q_{1}\right)\right], \\
c_{q}(\Lambda) & =\sum_{V: V \cap \Lambda \neq \varnothing}\left[W^{\Lambda}(V \mid q)-\frac{|V \cap \Lambda|}{|V|} W(V \mid q)\right] .
\end{aligned}
$$

Как будет ясно из условий теоремы 1 , величина $c_{q}(\Lambda)$ не влияет на термодинамические и корреляционные функции. Статистическую сумму (2) можно теперь записать в виде

$$
\Xi_{q}^{\Lambda}(V, H)=\sum_{\overrightarrow{\boldsymbol{\partial}} \in \gamma_{q}(V)} z_{q, \Lambda}^{V} z_{q}^{-V(\overrightarrow{\boldsymbol{\partial}})} e^{-H^{\Lambda}(\overrightarrow{\boldsymbol{\partial}})} \prod_{i \in l(\overrightarrow{\boldsymbol{\partial}})} z_{k_{i}(\overrightarrow{\boldsymbol{\partial}})}^{V_{i}(\overrightarrow{\boldsymbol{\partial}})}
$$

где $z_{q, \Lambda}(x):=e^{h_{q}^{\Lambda}(x)}$. Предельный гамильтониан границы, от которого зависят предельные статистические суммы (1), имеет вид

$$
\begin{gathered}
H(\overrightarrow{\boldsymbol{\partial}}):=H_{0}(\overrightarrow{\boldsymbol{\partial}})+H_{1}(\overrightarrow{\boldsymbol{\partial}}), \quad H_{0}(\overrightarrow{\boldsymbol{\partial}}):=\sum_{\overrightarrow{\boldsymbol{\Gamma}} \in \overrightarrow{\boldsymbol{\partial}}} H_{0}(\overrightarrow{\boldsymbol{\Gamma}}), \quad H_{1}(\overrightarrow{\boldsymbol{\partial}}):=\sum_{V} W_{1}(V \mid \overrightarrow{\boldsymbol{\partial}}), \\
W_{1}(V \mid \overrightarrow{\boldsymbol{\partial}}):=W(V \mid \overrightarrow{\boldsymbol{\partial}})-\sum_{q} \frac{\left|V_{q}(\overrightarrow{\boldsymbol{\partial}}) \cap V\right|}{|V|} W(V \mid q) .
\end{gathered}
$$

Физические условия, при которых построения для многофазных контурных моделей должны работать, ясны: энергетический барьер между различными невозмущенными фазами на микроуровне достаточно велик $\left(H_{0}(\overrightarrow{\boldsymbol{\Gamma}})\right.$ пропорционален “длине контура" с относительно большим коэффициентом пропорциональности), и взаимодействие контуров достаточно мало. Точная формулировка несколько длиннее.

Положим $W(\overrightarrow{\boldsymbol{\Gamma}}, \overrightarrow{\boldsymbol{\partial}} \backslash \overrightarrow{\boldsymbol{\Gamma}}):=H(\overrightarrow{\boldsymbol{\partial}})-H(\overrightarrow{\boldsymbol{\Gamma}})-H(\overrightarrow{\boldsymbol{\partial}} \backslash \overrightarrow{\boldsymbol{\Gamma}})$,

$$
\begin{aligned}
B(\overrightarrow{\boldsymbol{\Gamma}}) & :=\operatorname{Re}\left[-H_{1}(\overrightarrow{\boldsymbol{\Gamma}})\right]+\max _{\overrightarrow{\boldsymbol{\partial}}: \overrightarrow{\boldsymbol{\Gamma}} \in \min ^{0} \overrightarrow{\boldsymbol{\partial}}} \operatorname{Re}[-W(\overrightarrow{\boldsymbol{\Gamma}}, \overrightarrow{\boldsymbol{\partial}} \backslash \overrightarrow{\boldsymbol{\Gamma}})] \\
B^{(k)}(\overrightarrow{\boldsymbol{\Gamma}}) & :=\left|H_{1}^{(k)}(\overrightarrow{\boldsymbol{\Gamma}})\right|+\max _{\overrightarrow{\boldsymbol{\partial}}: \overrightarrow{\boldsymbol{\Gamma}} \in \min ^{0} \overrightarrow{\boldsymbol{\partial}}}\left|W^{(k)}(\overrightarrow{\boldsymbol{\Gamma}}, \overrightarrow{\boldsymbol{\partial}} \backslash \overrightarrow{\boldsymbol{\Gamma}})\right|
\end{aligned}
$$

где верхний индекс $(k)$ означает производную по параметрам, $k$ - мультииндекс. 
ЗАмЕчАНиЕ. В дальнейшем нам будут необходимы оценки производных термодинамических и некоторых вспомогательных величин. По ряду параметров оценки будут мажорантными; такие оценки допускают дифференцирование неравенств в следующем смысле. Пусть $f(z, t)=\sum_{X} c_{X}(t) z^{X}-$ ряд по положительным степеням $z\left(z, t\right.$ - векторы) и известен набор функций $\bar{c}_{X}(t)$ таких, что $\left|c_{X}(t)\right| \leqslant \bar{c}_{X}(t)$. Положим $\bar{f}(z, t):=\sum_{X} \bar{c}_{X}(t)|z|^{X}$. Тогда, очевидно, $|f(z, t)| \leqslant \bar{f}(z, t)$ и $\left|f_{z}^{(k)}(z, t)\right| \leqslant$ $\bar{f}_{|z|}^{(k)}(z, t)$. При этом мы будем говорить, что мажорантные оценки можно дифференцировать по $z$. Обозначим $g^{(k)}(t) \quad k$-ю производную по параметрам $t$, где $k-$ мультииндекс. Пусть известен набор функций $\bar{c}_{X}^{(k)}(t)$ такой, что $\left|c_{X}^{(k)}(t)\right| \leqslant \bar{c}_{X}^{(k)}(t)$, и $\bar{f}^{(k)}(z, t):=\sum_{X} \bar{c}_{X}^{(k)}(t)|z|^{X}$, тогда по построению $\left|f^{(k)}(z, t)\right| \leqslant \bar{f}^{(k)}(z, t)$. В этом случае также будем говорить, что исходное неравенство $|f| \leqslant \bar{f}$ допускает формальное дифференцирование, а $\bar{f}^{(k)}$ - формальная производная от $\bar{f}$. Весьма важно, что подобное формальное дифференцирование неравенств "выдерживает" ряд операций: $\left|\left(C_{1} f+C_{2} g\right)^{(k)}\right| \leqslant\left(\left|C_{1}\right| \bar{f}+\left|C_{2}\right| \bar{g}\right)^{(k)} ;\left|(f g)^{(k)}\right| \leqslant(\bar{f} \bar{g})^{(k)}$, где правая часть вычисляется по стандартным формулам дифференцирования; $\left|(f /(1+C g))^{(k)}\right| \leqslant(\bar{f} /(1-|C| \bar{g}))^{(k)}$; $\left|\left(e^{f}\right)^{\prime}\right| \leqslant \bar{f}^{\prime} e^{\operatorname{Re} f}=:\left(e^{\operatorname{Re} f}\right)^{\prime}$ и т.п. Использование подобной записи оценок более компактно, а также удобно при получении самих оценок. Особенно это удобно, когда оценка нужных функций является неявной функцией, например, производящей функцией числа “деревьев" типа $\bar{\omega}=z P(\bar{\omega}, t)$, где $P$ - сходящийся ряд с положительными коэффициентами по степеням $\bar{\omega}$. В этом случае оценки $\bar{\omega}$ сводятся к оценкам $P$. Легко проверить, что для получения оценок производных $\bar{\omega}(z, t)$ необходимо решить систему уравнений на $\bar{\omega}^{(k)}$, получаемую формальным дифференцированием функции $\bar{\omega}=z P(\bar{\omega}, t)$. Стоит отметить, что сначала можно найти оценку первой производной в виде $\bar{\omega}_{t}^{\prime}=\left(z_{t}^{\prime} P+z P_{t}^{\prime}\right) /\left(1-z P_{\bar{\omega}}^{\prime}\right)$, а затем искать оценки старших производных с помощью дифференцирования. Неравенства $z P\left(\bar{\omega}_{0}, t\right) \leqslant \bar{\omega}_{0}$ и $z P_{\bar{\omega}}^{\prime}\left(\bar{\omega}_{0}, t\right)<1$ для некоторого $\bar{\omega}_{0}$ обеспечивают конечность всех производных.

Пусть $b_{q}(q \in \Phi)$ - ограниченная последовательность комплексных чисел, для которой существует число $b_{\max }$ такое, что $\left|b_{\max }\right|=\sup \left|b_{q}\right|$, при этом для всех $q$ либо $b_{q}=b_{\max }$, либо $\left|b_{q}\right|<\left|b_{\max }\right|$. Пусть $p_{\max }:=\ln b_{\max }, p_{q}:=\ln b_{q}, \Delta p_{q}:=p_{q}-p_{\max }$, $b_{q}^{e}:=b_{q} / b_{\max }$. Вектор, составленный из $p_{q}$, будем обозначать как $\overrightarrow{\mathbf{p}}$ и называть вектором давлений фаз, $p_{q}$ - давление фазы $q, p_{\max }$ - давление в многофазной контурной модели $(1)^{5)}$.

Ослабленные условия $1-4$ на $W(V \mid \overrightarrow{\boldsymbol{\partial}})$ и $H_{0}(\overrightarrow{\boldsymbol{\Gamma}})$ теоремы II.4 (по существу, ослаблено лишь условие 3), которые применимы к рассмотрению поверхностных фаз, выглядят следующим образом.

Условие 1 (геометрические свойства границы). Существуют функиионалы $|\overrightarrow{\boldsymbol{\Gamma}}|$, $h(\overrightarrow{\boldsymbol{\Gamma}})^{6)}$, зависящие лищь от размерности пространства и удовлетворяющие неравенствам $\left|\partial V_{\overrightarrow{\boldsymbol{\Gamma}}}\right|+\left|V_{\overrightarrow{\boldsymbol{\Gamma}}}\right| \leqslant|\overrightarrow{\boldsymbol{\Gamma}}|, h(\overrightarrow{\boldsymbol{\Gamma}}) \geqslant 1$, где $\left|\partial V_{\overrightarrow{\boldsymbol{\Gamma}}}\right|-(\nu-1)$-мерный оббем геометрической границь $V,\left|V_{\overrightarrow{\boldsymbol{\Gamma}}}\right|-\nu$-мерный обвем $V_{\overrightarrow{\mathbf{\Gamma}}}$. Кроме того, для всех $V$, совпадающих либо с $V(\overrightarrow{\boldsymbol{\Gamma}})$, либо с $V_{i}(\overrightarrow{\boldsymbol{\Gamma}}), \sum\left|x_{1}-x_{2}\right| \leqslant C_{2} l|\partial V|$, если $x_{1} \in V, x_{2} \in \mathbb{Z}^{\nu} \backslash V,\left|x_{1}-x_{2}\right|=l$.

\footnotetext{
${ }^{5)}$ Ситуация, когда верхняя грань $p_{q}$ не достигается ни для какого $q$, не является математической экзотикой, а может иметь простой физический смысл, что будет показано в следующей работе.

6) В случае поверхностных фаз контур $\overrightarrow{\boldsymbol{\Gamma}}$ может обладать внутренней структурой, и тогда удобно ввести дополнительный геометрический функционал $h(\overrightarrow{\boldsymbol{\Gamma}})$.
} 
Условие 2 (локальность взаимодействия). Пусть $W^{\Lambda}(V \mid \vec{\partial})=W(V \mid \vec{\partial})$, $H_{0}^{\Lambda}(\overrightarrow{\boldsymbol{\Gamma}})=H_{0}(\overrightarrow{\boldsymbol{\Gamma}})$, если $V_{\overrightarrow{\boldsymbol{\Gamma}}}, V_{\overrightarrow{\boldsymbol{\partial}}} \subset \Lambda ; W(V \mid \overrightarrow{\boldsymbol{\partial}})=W(V \mid \overrightarrow{\boldsymbol{\partial}} \backslash \overrightarrow{\boldsymbol{\Gamma}})$, если $V_{\overrightarrow{\boldsymbol{\partial}} \backslash \overrightarrow{\boldsymbol{\Gamma}}} u V$ nрuнадлежат некоторой связной компоненте $R^{\nu} \backslash V_{\vec{\Gamma}}$.

УсловиЕ 3 (оценка взаимодействия контуров). Существуют функции $\bar{W}(V)$, удовлетворяющие следующим неравенствам для всех $\overrightarrow{\boldsymbol{\partial}}, \overrightarrow{\boldsymbol{\Gamma}} \in \min ^{0} \vec{\partial}$ :

$$
\max _{\substack{\overrightarrow{\boldsymbol{\partial}}_{1} \in \gamma: \overrightarrow{\boldsymbol{\partial}} \in \gamma\left(\overrightarrow{\boldsymbol{\partial}}_{1}\right), \min ^{0} \overrightarrow{\boldsymbol{\partial}} \subset \min ^{0} \overrightarrow{\boldsymbol{\partial}}_{1}}}\left|\sum_{\overrightarrow{\boldsymbol{\partial}}_{2} \subset \min ^{0} \overrightarrow{\boldsymbol{\partial}}}(-1)^{\left|\overrightarrow{\boldsymbol{\partial}}_{2}\right|} W\left(V \mid \overrightarrow{\boldsymbol{\partial}}_{1} \backslash \overrightarrow{\boldsymbol{\partial}}_{2}\right)\right| \leqslant 2^{|\overrightarrow{\boldsymbol{\partial}}|} h(\overrightarrow{\boldsymbol{\Gamma}}) \bar{W}(V), \quad|V| \geqslant 2,
$$

где $|\overrightarrow{\boldsymbol{\partial}}|$ - число контуров в $\vec{\partial}$. Для некоторого $\delta>0$ конечны величины

$$
\bar{W}_{2 x}(\delta, \mu)=\sum_{x}|x| \sum_{V \ni 0, x}(1+\delta)^{|V|} \bar{W}(V) .
$$

УсловиЕ 4 (Пайерлса-Пирогова-Синая). Для всех q справедливо неравенство

$$
|\overrightarrow{\boldsymbol{\Gamma}}|=S
$$

где $V_{q}^{\prime}(\overrightarrow{\boldsymbol{\Gamma}})=V(\overrightarrow{\boldsymbol{\Gamma}})$, если $q=k_{\mathrm{ex}}(\overrightarrow{\boldsymbol{\Gamma}})$ и равно $V_{q}(\overrightarrow{\boldsymbol{\Gamma}})$ в противоположном случае,

$$
\Delta \overrightarrow{\mathbf{p}} m_{1}(\overrightarrow{\boldsymbol{\Gamma}}):=\sum_{i} \Delta p_{q}\left|m_{1}\left(\partial V_{i}(\overrightarrow{\boldsymbol{\Gamma}})\right)\right|, \quad m_{1}(\partial)=\frac{|\partial|}{(2 \nu)} .
$$

УСловие 5 (гладкость по параметрам). Найдутся функции $\bar{W}^{(k)}(V), c_{k}(\mu) m a-$ кие, что при $k<k_{0}, k \neq 0$ справедливъ неравенства

$$
\begin{gathered}
\sum_{x}|x| \sum_{V \ni 0, x}(1+\delta)^{|V|} \bar{W}^{(k)}(V)<\infty \\
\sum_{\substack{\overrightarrow{\boldsymbol{\Gamma}}: \min _{1} V_{q}^{\prime}(\overrightarrow{\boldsymbol{\Gamma}})=0,|\overrightarrow{\boldsymbol{\Gamma}}|=S}} h(\overrightarrow{\boldsymbol{\Gamma}})\left|\exp \left\{-H_{0}(\overrightarrow{\boldsymbol{\Gamma}})-p_{\max }\left|V_{\overrightarrow{\boldsymbol{\Gamma}}}\right|+\Delta \overrightarrow{\mathbf{p}} m_{1}(\overrightarrow{\boldsymbol{\Gamma}})+B(\overrightarrow{\boldsymbol{\Gamma}})\right\}\right|^{(k)} \leqslant \\
\quad \leqslant c_{k}(\mu) S^{m(k)} \psi_{\text {cond }}^{S},
\end{gathered}
$$

где $m(k)$ - челое число, а такэе неравенство, получаемое формальным дифберенцированием неравенства (10).

Заметим, что чаще всего условие 5 не налагает дополнительных ограничений. Потенциалы $W(x \mid q)$ участвуют лишь в условии 4 .

Для каждой границы $\vec{\partial} \in \gamma_{q}$ определим геометрическую границу $\partial^{q}$ как границу области $V(\overrightarrow{\boldsymbol{\partial}}) \quad\left(\partial^{q}:=\partial V(\overrightarrow{\boldsymbol{\partial}})\right)$. Пусть для каждого $q$ заданы функционалы $F\left(\partial^{q}\right)$. Введем двухкомпонентные контурные модели с "внешним полем" при помощи статистических сумм (см. подробнее раздел I.4)

$$
\Xi_{+}(V, q):=\sum_{B: V(B) \subset V}\left(b_{q}^{e}\right)^{V_{\circ}(B)} F_{q}^{B}, \quad \Xi_{-}(V, q)=\sum_{B: V(B) \subset V}\left(b_{q}^{e}\right)^{V \backslash V_{\circ}(B)} F_{q}^{B},
$$


где $B$ - наборы непересекающихся контуров $\partial^{q}, V_{\text {о }}(B)$ - часть $V(B)$, охватываемая нечетным числом контуров из $B, F_{q}^{B}=\prod F\left(\partial^{q}\right), \partial^{q} \in B$. Положим

$$
\begin{gathered}
\varphi_{\partial \cup B}^{q}:=b^{V(\partial)}\left(-b^{V(\Gamma)}\right)^{B \backslash \min B}\left(b^{-V(\Gamma)}-b^{V(\Gamma)}\right)^{\min B}, \quad b=b_{q}^{e}, \\
\sigma_{q}(V):=\frac{\Xi_{-}(V, q)}{\Xi_{+}(V, q)}=\sum_{B: V(B) \subset V} F_{q}^{B} \varphi_{\partial V \cup B}^{q} \delta_{q}(\partial V \cup B), \\
\mathcal{F}\left(\partial^{q}\right):=F\left(\partial^{q}\right) \sigma_{q}\left(V\left(\partial^{q}\right)\right) .
\end{gathered}
$$

Введем давление $s_{q}$ для статсумм (14), а также функции $\delta_{q}(V), \Delta_{q}(V)$ и соответствующие кластерные разложения для $s_{q}, \ln \delta_{q}(V), \ln \Delta_{q}(V)\left(\right.$ см. раздел I.4 $\left.{ }^{7)}\right)$ :

$$
\begin{gathered}
s_{q}:=\lim _{V \uparrow Z^{\nu}} \frac{1}{|V|} \ln \Xi_{+}(V, q)=\sum_{B: \min _{1} B=0}^{0} \varphi_{q}^{0}(B), \\
\delta_{q}(\partial \cup B):=\Xi_{+}^{-1}(V(\partial), q) \prod_{i \in l(\partial \cup B)} \Xi_{+}\left(V_{i}, q\right)=\exp \left\{-\sum_{\substack{B_{1} \subset V(\partial), U_{0}(B, B) \neq 0}}^{0} \varphi_{q}^{0}(B)\right\}, \\
\Delta_{q}(V):=e^{-s_{q}|V|} \Xi_{+}(V, q)=\exp \left\{-\sum_{V(B) \not \subset V}^{0} \varphi_{q}^{0}(B) \frac{|V \cap V(B)|}{|V(B)|}\right\},
\end{gathered}
$$

где $\varphi_{q}^{0}(B)$ - функция Урселла, построенная по функциям $\mathcal{F}_{q}^{B} e^{-U_{0}(B)}, U_{0}(B)$ - потенциал "твердых сердцевин" контуров $B$. Для $\vec{\partial} \in \gamma_{q}$, используя предыдущие функции, введем

$$
\Delta(\overrightarrow{\boldsymbol{\partial}}):=\Delta_{q}^{-1}(V(\overrightarrow{\boldsymbol{\partial}})) \prod_{i \in l(\overrightarrow{\boldsymbol{\partial}})} \Delta_{k_{i}}\left(V_{i}(\overrightarrow{\boldsymbol{\partial}})\right), \quad \sigma(\overrightarrow{\boldsymbol{\partial}}):=\prod_{i \in l(\overrightarrow{\boldsymbol{\partial}})} \sigma_{k_{i}}\left(V_{i}(\overrightarrow{\boldsymbol{\partial}})\right) .
$$

Уравнение состояния и контурные уравнения имеют вид (см. раздел I.5)

$$
\begin{gathered}
p_{q}=h_{q}+s_{q}\left(\mathcal{F}_{q}\right), \\
F\left(\partial^{q}\right)=\sum_{\vec{\partial}: \overrightarrow{\boldsymbol{\partial}}^{\mathrm{ex}}=\partial^{q}} b_{\max }^{-V_{\overrightarrow{\vec{x}}}} \varphi_{\vec{\partial}} \Delta(\overrightarrow{\boldsymbol{\partial}}) \sigma(\overrightarrow{\boldsymbol{\partial}}), \\
\sigma_{q}(V)=\sum_{B: V(B) \subset V} F_{q}^{B} \varphi_{\partial V \cup B}^{q} \delta_{q}(\partial V \cup B), \\
\mathcal{F}\left(\partial^{q}\right)=F\left(\partial^{q}\right) \sigma_{q}\left(V\left(\partial^{q}\right)\right) .
\end{gathered}
$$

Запишем уравнение состояния как отображение $\overrightarrow{\mathbf{p}} \rightarrow \overrightarrow{\mathbf{h}}$ :

$$
\overrightarrow{\mathbf{h}}(\overrightarrow{\mathbf{p}}, \mu):=\overrightarrow{\mathbf{p}}-\overrightarrow{\mathbf{s}}(\overrightarrow{\mathbf{p}}, \mu),
$$

а контурные уравнения - в векторной форме

$$
\overrightarrow{\mathcal{F}}=\overrightarrow{\boldsymbol{\Upsilon}}\left(\overrightarrow{\mathcal{F}}, \Delta \overrightarrow{\mathbf{p}}, e^{-p_{\max } V_{\vec{\partial}}} \varphi_{\vec{\partial}}\right)
$$

\footnotetext{
7)Если в статсумме идет суммирование по непересекающимся контурам, то при формальном переразложении логарифма статсуммы и величин типа $s_{q}$ суммирование ведется по произвольным наборам контуров $X$, среди которых могут быть и повторяющиеся контуры. Для повторяющихся контуров в соответствующих разложениях необходимо добавить в знаменатель факториалы числа повторяющихся контуров. Сумма $\sum^{0}$ содержит в себе эти сомножители.
} 
Нам удобно обозначить сужение вектора $\overrightarrow{\mathbf{d}}$ на подпространство векторов с номерами компонент из $G \subset \Phi$ как $\left.\overrightarrow{\mathbf{d}}\right|_{G}$, неравенства на векторы понимать как покомпонентные. Пусть вектор $\left.e^{\overrightarrow{\mathbf{d}}}\right|_{G}$ имеет компоненты, равные $e^{d_{q}}, q \in G$. Введем области в $\mathbb{C}$ вида

$$
\bar{S}_{1}(a)=\left\{b:|b|^{2}+a|b-1| \leqslant 1\right\}, \quad a<1 ;
$$

пусть $S_{1}(a)$ - множество внутренних точек $\bar{S}_{1}(a)$. Область $S_{1}(a)$ содержит комплексную окрестность отрезка $(0,1)$.

Определим комплексные области

$$
\begin{aligned}
\Im & :=\left\{(\overrightarrow{\mathbf{h}}, \mu): \psi_{\text {cond }}(\overrightarrow{\mathbf{h}}) \leqslant \psi_{0}, C \psi_{0}^{2 \nu} \bar{W}_{2 x}\left(C \psi_{0}^{2 \nu}, \mu\right) \leqslant \psi_{1},\right. \\
\left.\bar{W}_{2 x}^{(k)}\left(C \psi_{0}^{2 \nu}, \mu\right)<\infty, c_{k}(\mu)<\infty\right\}, & \\
\mathcal{M}:=\{\mu:(\overrightarrow{\mathbf{h}}(\mu), \mu) \in \Im\} &
\end{aligned}
$$

соответствующие вещественные области обозначим как $\Im_{\mathbb{R}}, \mathcal{M}_{\mathbb{R}}$. Напомним, что $\left|V_{\overrightarrow{\boldsymbol{\Gamma}}}\right|$ - это $\nu$-мерный объем носителя контура; для "тонких" контуров, у которых носитель имеет размерность $\nu-1$, он равен нулю. Следовательно, в этом случае в (21) внешнее поле отсутствует. Вследствие "почти" однородности исходных статсумм относительно растяжения вектора активностей фаз (см. (1), а также раздел I.4) величину $\max \operatorname{Re} h_{q}$ можно считать равной нулю. Поэтому при общем определении носителя границы в приложениях внешние поля в (21) фактически произвольны.

Определим следующие плоские области в расширенном пространстве параметров:

$$
\begin{aligned}
\partial_{G \wp_{\overrightarrow{\mathbf{0}}}}^{\mathbb{C}}:=\left\{(\overrightarrow{\mathbf{p}}, \mu): \overrightarrow{\mathbf{p}} \in \mathbb{C}^{\Phi},\left.\overrightarrow{\mathbf{p}}\right|_{G}=\left.p_{\max } \overrightarrow{\mathbf{1}}\right|_{G}, e^{\Delta p_{q}} \in S_{1}\left(C \psi_{0}^{2 \nu}\right) \forall q \in \Phi \backslash G,\right. \\
\psi_{\text {cond }}(\overrightarrow{\mathbf{p}}) \leqslant \psi_{0}, C \psi_{0}^{2 \nu} \bar{W}_{2 x}\left(C \psi_{0}^{2 \nu}, \mu\right) \leqslant \psi_{1}, \\
\left.\bar{W}_{2 x}^{(k)}\left(C \psi_{0}^{2 \nu}, \mu\right)<\infty, c_{k}(\mu)<\infty\right\}, \\
\wp_{\overrightarrow{\mathbf{0}}}^{\mathbb{C}}:=\bigcup_{G \subset \Phi} \partial_{G \wp_{\overrightarrow{\mathbf{0}}}} \mathbb{C},
\end{aligned}
$$

где $C$ - константа, зависящая лишь от $\nu$. Заметим, что вещественная часть $\wp_{\overrightarrow{0}}^{\mathbb{C}}$ содержит $\mathbb{R}^{\Phi} \times \mathcal{M} \subset \wp_{\overrightarrow{\mathbf{0}}}^{\mathbb{C}}$ (вещественные давления произвольны), если вместо (11) мы введем определение

$$
\psi_{\text {cond }}(\overrightarrow{\mathbf{p}}):=\inf _{q, S}\left\{\sum_{\substack{\overrightarrow{\boldsymbol{\Gamma}}: \min _{1} V_{q}^{\prime}(\overrightarrow{\boldsymbol{\Gamma}})=0,|\overrightarrow{\boldsymbol{\Gamma}}|=S}} h(\overrightarrow{\boldsymbol{\Gamma}})\left|e^{-H_{0}(\overrightarrow{\boldsymbol{\Gamma}})-p_{\max }\left|V_{\overrightarrow{\boldsymbol{\Gamma}}}\right|+B(\overrightarrow{\boldsymbol{\Gamma}})}\right|\right\}^{1 / S} .
$$

Теорема 1. Пусть выполнены условия 1-5 при замене $W(V \mid \overrightarrow{\boldsymbol{\partial}})$ и $H_{0}(\overrightarrow{\boldsymbol{\Gamma}})$ на $W^{\Lambda}(V \mid \overrightarrow{\boldsymbol{\partial}})$ и $H_{0}^{\Lambda}(\overrightarrow{\boldsymbol{\Gamma}})$, начиная с некоторого $\Lambda$ при $\Lambda \uparrow \mathbb{Z}^{\nu}$. Тогда существуют малье константы $\psi_{0}, \psi_{1}$ такие, что области $\wp_{\overrightarrow{\mathbf{0}}}^{\mathbb{C}}, \Im_{\overrightarrow{\mathbf{0}}}^{\mathbb{C}}:=\overrightarrow{\mathbf{h}}\left(\wp_{\overrightarrow{\mathbf{0}}}^{\mathbb{C}}\right), \mathcal{M}_{\overrightarrow{\mathbf{0}}}:=\{\mu \in \mathcal{M}: \overrightarrow{\mathbf{h}}(\mu) \in$ $\left.\Im_{\overrightarrow{0}}^{C}\right\}$ являются областями фазовых переходов первого рода (в этих областях по определению выполнены теоремы I.4- I.7). В частности, верны следующие утверждения. 
1. Контурные уравнения имеют решения в области $\wp_{\overrightarrow{\mathbf{0}}}^{\mathbb{C}}, u$ в этой области определено непрерывное отображение $\overrightarrow{\mathbf{h}}(\overrightarrow{\mathbf{p}}, \mu)$. При использовании определения (23) подобласть $\wp_{\overrightarrow{\mathbf{0}}}^{\mathbb{C}}$ содержит $\mathbb{R}^{\Phi} \times \mathcal{M}$, и в ней вектор давлений произволен.

2. Решение контурных уравнений и бункиия $\overrightarrow{\mathbf{h}}(\overrightarrow{\mathbf{p}}, \mu)$ разлагаются в сходящиеся (равномерно по $\overrightarrow{\mathbf{p}}$ ) ряды по степеням $\varphi_{\vec{\partial}}$ в $\wp_{\overrightarrow{\mathbf{D}}}^{\mathbb{C}}$; эти функции являются гладкими (аналитическими) в каждом страте $\partial_{G \varnothing \overrightarrow{\mathbf{0}}}^{\mathbb{C}}$, если гладки (аналитичны) в области $\mathcal{M}$ величины $W(V \mid \overrightarrow{\boldsymbol{\partial}})$ u $e^{-H_{0}(\overrightarrow{\boldsymbol{\Gamma}})}$. Значения производных продолжаются до непрерывных в замыкании страта $\partial_{G} \wp_{\overrightarrow{\mathbf{0}}}^{\mathbb{C}}$, предельные значения производных гладки (аналитичны) в каждом из стратов $\partial_{G_{1}} \wp \mathbb{C}, G \subset G_{1}$.

3. $В \partial_{G \wp} \wp_{\overrightarrow{\mathbf{0}}}^{\mathbb{C}}$ норма $\partial \overrightarrow{\mathbf{s}}(\overrightarrow{\mathbf{p}}, \mu) / \partial \overrightarrow{\mathbf{p}}$ менъше единицы, отображение $\overrightarrow{\mathbf{h}}(\overrightarrow{\mathbf{p}}, \mu)$ взаимно одназначно при фиксированном $\mu$ (см. теорему I.5).

4. Область $\Im_{\overrightarrow{0}}^{\mathbb{C}}$ содержит $\mathbb{R}^{\Phi} \times \mathcal{M}$. Страты $\partial_{G} \Im_{\overrightarrow{0}}^{\mathbb{C}}-$ гладкие (комплексные) многообразия комплексной коразмерности $|G|-1$. Обратное отображение $\overrightarrow{\mathbf{p}}(\overrightarrow{\mathbf{h}}, \mu)$ является непрерывным и гладким (аналитичным) в каждом страте $\partial_{G} \Im_{\overrightarrow{\mathbf{0}}}^{\mathbb{C}}$. Значения производных продолжсатся до непрерывных в замыкании страта $\partial_{G} \Im_{\overrightarrow{\mathbf{0}}}^{\mathbb{C}}$, предельные значения производных гладки (аналитичны) в каждом из стратов $\partial_{G_{1}} \Im_{\overrightarrow{\mathbf{0}}}^{\mathbb{C}}, G \subset G_{1}$.

5. Давление в модели определяется функиией $p_{\max }(\overrightarrow{\mathbf{h}}, \mu)$ и обладает теми же дифференииальньми свойствами, что и функция $\overrightarrow{\mathbf{p}}(\overrightarrow{\mathbf{h}}, \mu)$. Если $\overrightarrow{\mathbf{h}}=\overrightarrow{\mathbf{h}}(\mu)$, то зависимость от параметров $\mu$ вектора давления и его дифференциальные свойства определяются функиией $\overrightarrow{\mathbf{p}}(\mu):=\overrightarrow{\mathbf{p}}(\overrightarrow{\mathbf{h}}(\mu), \mu)$ и наследуются из свойств $\overrightarrow{\mathbf{p}}(\overrightarrow{\mathbf{h}}, \mu)$. Вектор давлений $\overrightarrow{\mathbf{p}}(\mu)$ определен в области $\mathcal{M}_{\overrightarrow{\mathbf{0}}} \supset \mathcal{M}_{\mathbb{R}}$. Каждыи страт $\partial_{G} \mathcal{M}_{\overrightarrow{\mathbf{0}}}:=\{\mu \in$ $\left.\mathcal{M}_{\overrightarrow{\mathbf{0}}}: \overrightarrow{\mathbf{h}}(\mu) \in \partial_{G} \Im_{\overrightarrow{\mathbf{0}}}^{C}\right\}$ является вещественно-аналитическим множеством.

6. Для статистических сумм (1) справедливы следующие представления:

$$
\Xi_{q}(V, H)=e^{p_{\max }|V|} \Delta_{q}(V) \sigma_{q}(V)
$$

причем $\left|\sigma_{q}(V)\right| \leqslant 1$. Для устойчивых фаз, где $p_{q}=p_{\max } u \sigma_{q}(V)=1$,

$$
\Xi_{q}(V, H)=e^{p_{\max }|V|} \Delta_{q}(V),
$$

причем $\left|\ln \Xi_{q}(V, H)-p_{\max }\right| V|| \leqslant C \psi_{0}^{2 \nu}|\partial V|$.

7. При пересечении страта фазовой диаграммы в пространстве параметров разрывна одна из производных давления по $\overrightarrow{\mathbf{h}}$. Гиббсовское состояние, построенное по граничному условию

$$
q \in G(\mu):=\left\{q \in \Phi: p_{q}(\mu)=p_{\max }(\mu)\right\},
$$

является малым возмущением фазы q (см. теорему I.5).

Фазы, принадлежащие множеству $\Phi \backslash G(\mu)$, будем называть неустойчивыми. Следующая теорема удобна для анализа фазовых диаграмм.

ТЕОРема 2 (критерий устойчивости фаз). В области фазовых переходов первого рода для неустойчивых фаз $q \in \Phi \backslash G(\mu)$ справедливо неравенство

$$
\left|\Xi_{q}(V, H) e^{-p_{\max }|V|}\right| \leqslant\left[C_{2} \psi_{0}\right]^{|\partial V| / 2},
$$



если $|V|-C_{3}|\partial V| \geqslant-\left[\ln \left(C_{2} \psi_{0}\right)\right] /\left(2\left|p_{q}-p_{\max }\right|\right), V-\kappa у б$.

Для устойчивых фаз $q \in G(\mu)$ справедливо неравенство

$$
\left|\Xi_{q}(V, H) e^{-p_{\max }|V|}\right| \geqslant e^{-\bar{\omega}|\partial V|}, \quad \bar{\omega}=C_{1} \psi_{0}^{2 \nu}
$$

Из качественных соображений ясно, что для неустойчивых $q$ при достаточно больших объемах куба $V$ основной вклад в статсумму вносят конфигурации с контуром, близким к границе куба, объем, ограниченный этим контуром, примерно равен $|V|-C|\partial V|$, и он разделяет неустойчивую фазу $q$ и некоторую устойчивую фазу $q_{1}$. Теорема 2 дает грубую оценку соответствующего поверхностного натяжения, а также грубую оценку величины объемов, начиная с которых конфигурация с большим контуром, содержащим внутри себя устойчивую фазу, дает основной вклад в статсумму. Теорема 2 доказана в приложении.

Напомним, что геометрическая граница д области $V(\overrightarrow{\boldsymbol{\partial}})$, вообще говоря, несвязна; обозначим через Г геометрическую границу области $V(\overrightarrow{\boldsymbol{\Gamma}}), \overrightarrow{\boldsymbol{\Gamma}} \in \vec{\delta}$. Доказательство теоремы 1 сводится (см. раздел I.5) к оценкам сумм

$$
\sum_{\overrightarrow{\boldsymbol{\partial}}: \partial V(\overrightarrow{\boldsymbol{\partial}}) \stackrel{*}{\cap} 0 \neq \varnothing}\left|z_{0}^{\overrightarrow{\boldsymbol{\partial}}} \varphi([\overrightarrow{\boldsymbol{\partial}}])\right|, \quad z_{0}^{\vec{\partial}}:=\prod_{\overrightarrow{\boldsymbol{\Gamma}} \in \overrightarrow{\boldsymbol{\partial}}} z_{0}(\overrightarrow{\boldsymbol{\Gamma}}),
$$

а сумма по границам $\partial \stackrel{*}{\cap} 0 \neq \varnothing$ имеет следующий вид (см. формулу (I.28)):

$$
\begin{gathered}
\sum_{\Gamma: \min _{1} \Gamma=0}\left\{C_{1}|\Gamma| \sum_{\partial \ni \Gamma} \bar{\omega}(\partial)+C_{2} \sum_{\substack{\partial: \Gamma_{0} \subset V(\Gamma), \Gamma \in \partial}} \bar{\omega}(\partial)+C_{3} \sum_{\Gamma_{1}, \partial: \partial \ni \Gamma, \Gamma_{1}} d\left(\Gamma, \Gamma_{1}\right) \bar{\omega}(\partial)\right\} \\
\bar{W}_{2}(\delta, \mu):=\sum_{x} \bar{W}_{2}(x, \delta, \mu),
\end{gathered}
$$

где $d\left(\Gamma_{1}, \Gamma_{2}\right)$ - расстояние между $\Gamma_{1}$ и $\Gamma_{2}, \Gamma_{0}$ - граница единичного куба. Введем несколько вспомогательных функций. Пусть

$$
\begin{gathered}
\bar{W}_{2}(x, \delta, \mu):=\sum_{V \ni 0, x}(1+\delta)^{|V|} W(V) \\
\bar{W}_{2 x}(\delta, \mu):=\sum_{x}|x| \sum_{V \ni 0, x}(1+\delta)^{|V|} W(V) \\
z(\overrightarrow{\boldsymbol{\Gamma}}):=\mid e^{-H_{0}(\overrightarrow{\boldsymbol{\Gamma}})-H_{1}(\overrightarrow{\boldsymbol{\Gamma}})-\max _{q} \operatorname{Re} h_{q}\left|V_{\overrightarrow{\boldsymbol{\partial}}}\right|+\Delta \overrightarrow{\mathbf{h}} m_{1}(\overrightarrow{\boldsymbol{\partial}})+B(\overrightarrow{\boldsymbol{\Gamma}}) \mid \times} \\
\times e^{\bar{\omega}\left|\partial V_{\overrightarrow{\boldsymbol{\partial}}}\right|+\bar{\omega}|\partial V(\overrightarrow{\boldsymbol{\partial}})|+\bar{\mu}_{V}|V(\overrightarrow{\boldsymbol{\Gamma}})|+\bar{\mu}_{\Gamma}|\overrightarrow{\boldsymbol{\Gamma}}|_{1}+C_{5} \bar{\omega}|\overrightarrow{\boldsymbol{\Gamma}}|}
\end{gathered}
$$

где $\bar{\mu}_{V}, \bar{\mu}_{\Gamma}-$ вспомогательные параметры, которые в случае отсутствия производных или после необходимых дифференцирований будут положены равными нулю, $B(\overrightarrow{\boldsymbol{\Gamma}})$ 
задано в (9). Введем также функции

$$
\begin{aligned}
& g_{i, j}(\lambda, \mu):=\max _{q} \sum_{\overrightarrow{\boldsymbol{\Gamma}}: 0 \in V_{q}^{\prime}(\overrightarrow{\boldsymbol{\Gamma}})} h(\overrightarrow{\boldsymbol{\Gamma}})|\overrightarrow{\boldsymbol{\Gamma}}|^{i}|V(\overrightarrow{\boldsymbol{\Gamma}})|^{j}|z(\overrightarrow{\boldsymbol{\Gamma}})| e^{\lambda|\overrightarrow{\boldsymbol{\Gamma}}|}, \\
& g_{i, j, x}(\lambda, \mu):=\max _{q} \sum_{x}|x| \sum_{\substack{\overrightarrow{\boldsymbol{\Gamma}}: \\
x \in V_{q}^{\prime}(\overrightarrow{\boldsymbol{\Gamma}})}} h(\overrightarrow{\boldsymbol{\Gamma}})|\overrightarrow{\boldsymbol{\Gamma}}|^{i}|V(\overrightarrow{\boldsymbol{\Gamma}})|^{j-2}|z(\overrightarrow{\boldsymbol{\Gamma}})| e^{\lambda|\overrightarrow{\boldsymbol{\Gamma}}|}, \\
& \widetilde{a}(\lambda, \mu):=g_{0,2}(\lambda, \mu) e^{g_{0,2}(\lambda, \mu)}, \quad \kappa(\lambda, \mu):=\tilde{a}(\lambda, \mu) \bar{W}_{2 x}(\tilde{a}, \mu), \\
& P_{2 x}(\lambda, \mu):=\frac{\tilde{a}(\lambda, \mu)\left(g_{0,2, x}(\lambda, \mu) \bar{W}_{2}(\tilde{a}, \mu)+g_{0,2}(\lambda, \mu) \bar{W}_{2 x}(\tilde{a}, \mu)\right)}{\left(1-g_{0,2}(\lambda, \mu) \bar{W}_{2}(\tilde{a}, \mu)\right)^{2}} .
\end{aligned}
$$

Отметим, что все величины в (30) малы, если малы $\psi_{0}, \psi_{1}$ в определении $\mathcal{M}$ (см. (21)) и $i, j$ ограничены. Определим стандартно формальные частные производные от введенных функций по параметрам модели $\mu$ и $\lambda$. Положим теперь

$$
\begin{aligned}
\mathcal{M}:= & \bigcup_{\lambda>0}\left\{\mu: \lambda \geqslant \kappa(\lambda, \mu), \kappa(\lambda, \mu)_{\lambda}^{\prime}<1, g_{0,2} \bar{W}_{2}(\tilde{a}, \mu)<1,\right. \\
& \left.\bar{W}_{2 x}^{(k)}<\infty, c_{k}(\mu)<\infty\right\}
\end{aligned}
$$

и аналогично определим $\Im$. Если в определениях (30) и (31) внешние поля заменить на давления, то ниже в теореме 3 мы найдем область в пространстве давлений.

Теорема 1 есть следствие следующей теоремы, в которой даны оценки сумм (27).

ТЕОРема 3 (оценки сумм функций Урселла гамильтониана границы). $B$ области (31) существует решение уравнения $\lambda=\kappa(\lambda, \mu)$, причем если для некоторого $\lambda_{0}$ выполнено неравенство $\kappa\left(\lambda_{0}, \mu\right) \leqslant \lambda_{0}$, то $\lambda(\mu) \leqslant \kappa\left(\lambda_{0}, \mu\right) \leqslant \lambda_{0}$. Справедливъ следующие мажсорантные оценки относительно $z_{0}(\overrightarrow{\boldsymbol{\Gamma}}):=z(\overrightarrow{\boldsymbol{\Gamma}}) e^{-B(\overrightarrow{\boldsymbol{\Gamma}})}$ :

$$
\max _{q} \sum_{\overrightarrow{\boldsymbol{\Gamma}}: 0 \in V_{q}^{\prime}(\overrightarrow{\boldsymbol{\Gamma}})}|\overrightarrow{\boldsymbol{\Gamma}}|_{1}^{i}|V(\overrightarrow{\boldsymbol{\Gamma}})|^{j} \sum_{\overrightarrow{\boldsymbol{\partial}}: \overrightarrow{\boldsymbol{\Gamma}} \in \overrightarrow{\boldsymbol{\partial}}}\left|z_{0}(\overrightarrow{\boldsymbol{\Gamma}})^{\overrightarrow{\boldsymbol{\partial}}} \varphi([\overrightarrow{\boldsymbol{\partial}}])\right| \leqslant g_{i, j}(\lambda(\mu), \mu),
$$

а также соответствующие неравенства на производные, в которых формальные производные от правой части надо находить, учитывая неравенство

$$
\lambda^{\prime}(\mu):=\frac{\kappa_{\mu}^{\prime}(\lambda(\mu), \mu)}{1-\kappa_{\lambda}^{\prime}(\lambda(\mu), \mu)} \leqslant \frac{\kappa_{\mu}^{\prime}\left(\lambda_{0}, \mu\right)}{1-\kappa_{\lambda}^{\prime}\left(\lambda_{0}, \mu\right)} .
$$

Верно также неравенство

$$
\sum_{\substack{\overrightarrow{\boldsymbol{\Gamma}}_{1}, \overrightarrow{\boldsymbol{\Gamma}}_{2} \in \gamma_{q}, \min _{1} V\left(\overrightarrow{\boldsymbol{\Gamma}}_{1}\right)=0}} d\left(\overrightarrow{\boldsymbol{\Gamma}}_{1}, \overrightarrow{\boldsymbol{\Gamma}}_{2}\right) \sum_{\overrightarrow{\boldsymbol{\partial}} \in \gamma_{q}: \overrightarrow{\boldsymbol{\Gamma}}_{1}, \overrightarrow{\boldsymbol{\Gamma}}_{2} \in \operatorname{ex} \overrightarrow{\boldsymbol{\partial}}}\left|z_{0}(\overrightarrow{\boldsymbol{\Gamma}})^{\overrightarrow{\boldsymbol{\partial}}} \varphi([\overrightarrow{\boldsymbol{\partial}}])\right| \leqslant P_{2 x}(\lambda(\mu), \mu)
$$

здесъ ех $\overrightarrow{\boldsymbol{\partial}}$ - внешние контуръ $\overrightarrow{\boldsymbol{\partial}}$. Кроме того, в случае $h(\overrightarrow{\boldsymbol{\Gamma}})=1$ верно неравенство $B(\overrightarrow{\boldsymbol{\Gamma}}) \leqslant r(\mu)|\overrightarrow{\boldsymbol{\Gamma}}|(c \mathcal{} .(9))$, дде $r(\mu)=C_{4} \bar{W}_{2 x}(0, \mu), r^{(k)}(\mu)=C_{4} \bar{W}_{2 x}^{(k)}(0, \mu)$.

Для того чтобы показать справедливость теоремы 1 , нам достаточно доказать (см. доказательство теорем I.4 и I.5), что неравенства $\bar{\omega}_{p}^{\prime}<1, \bar{\omega}_{\mu}^{\prime}<\infty$ и неравенство (I.60) 
верны для достаточно малых $\psi_{0}, \psi_{1}$ в (21). В неравенстве (І.60) увеличим левую часть, положив $\bar{\omega}_{q}=\bar{\omega}$ для всех $q$ и заменив $b_{q}^{e}=e^{\left(p_{q}-p_{\max }\right)}$ на $e^{h_{q}-\max \operatorname{Re} h_{q}+2 \bar{\omega}}$ (таким образом, мы проводим оценки в пространстве $\Im$ или $\mathcal{M})$, тогда получим под знаком суммы в левой части (І.60) следующее выражение $\left(\Delta \overrightarrow{\mathbf{h}} m_{1}(\overrightarrow{\boldsymbol{\partial}})\right.$ определено в $\left.(12)\right)$ :

$$
\left.\left[e^{-H_{0}(\overrightarrow{\boldsymbol{\Gamma}})-H_{1}(\overrightarrow{\boldsymbol{\Gamma}})}\right]\right]^{\overrightarrow{\boldsymbol{\partial}}} \varphi([\overrightarrow{\boldsymbol{\partial}}]) e^{-\max \operatorname{Re} h_{q}\left|V_{\overrightarrow{\boldsymbol{\partial}}}\right|+\bar{\omega}\left|\partial V_{\overrightarrow{\boldsymbol{\partial}}}\right|+\bar{\omega}|\partial V(\overrightarrow{\boldsymbol{\partial}})|+\Delta \overrightarrow{\mathbf{h}} m_{1}(\overrightarrow{\boldsymbol{\partial}})} .
$$

Таким образом, неравенство (І.60) можно записать в виде (условия суммирования определены в (28))

$$
\sum_{\vec{\partial}: \vec{\partial}^{\mathrm{ex}}=\partial^{q} \stackrel{\cap}{\cap}_{0 \neq \varnothing}}\left|z_{0}(\overrightarrow{\boldsymbol{\Gamma}})^{\vec{\partial}} \varphi([\overrightarrow{\boldsymbol{\partial}}])\right| \leqslant \bar{\omega} .
$$

Введем функцию $Q(\lambda, \mu, \bar{\omega}):=C_{1} g_{1,0}+C_{2} g_{0,1}+C_{3} P_{2 x}$; тогда, как это вытекает из теоремы 3 , неравенство (34) запишется в виде

$$
Q(\lambda(\mu, \bar{\omega}), \mu, \bar{\omega}) \leqslant \bar{\omega} .
$$

Определим стандартно формальные производные по параметрам, а также "полные" производные $Q_{\Gamma}:=Q_{\lambda}^{\prime} \lambda_{\Gamma}^{\prime}+Q_{\Gamma}^{\prime}, \tilde{a}_{V}:=\tilde{a}_{\lambda}^{\prime} \lambda_{V}^{\prime}+\tilde{a}_{V}^{\prime}$. Аналогично определим $Q_{V}, Q_{\mu}$, $\tilde{a}_{\Gamma}, \tilde{a}_{\mu}$, где $Q_{\lambda}^{\prime}, Q_{\mu}^{\prime}, Q_{V}^{\prime}, Q_{\Gamma}^{\prime}-$ частные формальные производные по $\lambda, \mu, \bar{\mu}_{V}, \bar{\mu}_{\Gamma}$ от $Q$. Сравнивая определения (I.56)-(I.59) и приведенные здесь, находим, что

$$
\begin{gathered}
a \leqslant \tilde{a}, \quad a_{1} \leqslant C_{6} \tilde{a}_{\Gamma}, \quad \bar{\omega}_{1} \leqslant C_{6} Q_{\Gamma}, \quad a_{2} \leqslant 2 \tilde{a}_{V}, \quad \bar{\omega}_{1} \leqslant 2 C_{6} Q_{\Gamma}, \quad \bar{\omega}_{2} \leqslant 2 Q_{V}, \\
a_{3,4} \leqslant \tilde{a}_{V}, \quad \bar{\omega}_{3,4} \leqslant Q_{V}, \quad a_{\mu} \leqslant \tilde{a}_{\mu}, \quad \bar{\omega}_{\mu} \leqslant Q_{\mu} .
\end{gathered}
$$

Из предыдущих неравенств и определений (I.56)-(I.59) находим также, что

$$
\bar{\omega}_{p}^{\prime} \leqslant \frac{Q_{V}\left(3+4 \tilde{a}_{V}\right)}{1-C_{6} Q_{\Gamma}-\tilde{a}_{V}-C_{6} Q_{\Gamma} \tilde{a}_{V}}, \quad \bar{\omega}_{\mu}^{\prime} \leqslant \frac{Q_{\mu}+Q_{V} \tilde{a}_{\mu}}{1-C_{6} Q_{\Gamma}-\tilde{a}_{V}-C_{6} Q_{\Gamma} \tilde{a}_{V}} .
$$

Заметим, что из оценок $|V(\overrightarrow{\boldsymbol{\Gamma}})| \leqslant C_{6}|\overrightarrow{\boldsymbol{\Gamma}}|^{\nu /(\nu-1)}$ и $|x| \leqslant|\overrightarrow{\boldsymbol{\Gamma}}|$ для $0, x \in V(\overrightarrow{\boldsymbol{\Gamma}})$ вытекает, что $g_{i, j} \leqslant C_{7} g_{i+2 j, 0}, g_{i, j, x} \leqslant C_{8} g_{i+1, j} \leqslant C_{9} g_{i+2 j+1,0}$. Из (27)-(28) и теоремы 3 получаем, что неравенство (34) следует из $Q(\lambda(\mu, \bar{\omega}), \mu, \bar{\omega}) \leqslant \bar{\omega}$. Действительно, пусть $\lambda_{0}=1, \bar{\omega}=1$. Тогда при достаточно малом $g_{5,0}$ малы $Q(1, \mu, 1)$ и $\tilde{a}$, а при достаточно малых $g_{7,0}$ и $g_{7,0}^{\prime}{ }_{\mu}$ малы величины $\bar{\omega}_{p}^{\prime}, \bar{\omega}_{\mu}^{\prime}$. Заметим, что производные высших порядков могут быть найдены из (36) формальным дифференцированием. Таким образом, повышение порядка производной не налагает дополнительных ограничений на область $\mathcal{M}$. Теорема 1 доказана.

Из доказательства теоремы следует, что можно определить области $\mathcal{M}, \Im$ точнее:

$$
\begin{aligned}
\mathcal{M}:= & \left\{\mu: \exists \lambda, \bar{\omega}: \kappa(\lambda, \mu) \leqslant \lambda, \kappa_{\lambda}^{\prime}(\lambda, \mu)<1, Q(\lambda, \mu, \bar{\omega}) \leqslant \bar{\omega}, g_{0,2} \bar{W}_{2}(\tilde{a}, \mu)<1,\right. \\
& \left.\bar{\omega}_{p}^{\prime}<1, \bar{W}_{2 x}^{(k)}(\tilde{a}, \mu)<\infty, c_{k}(\mu)<\infty, k<k_{0}\right\} .
\end{aligned}
$$

Отсюда видно, что как $\lambda$, так и $\bar{\omega}$ можно выбрать в виде $C \psi_{0}^{2 \nu}$.

Область $\mathcal{M}$ из (21) при достаточно малых $\psi_{0}, \psi_{1}$ лежит внутри области $\mathcal{M}(37)$, поэтому мы обозначаем расширенную область той же буквой. 


\section{3. ДОКАЗАТЕЛЬСТВО ТЕОРЕМЫ 3}

Для доказательства теоремы 3 и, следовательно, теоремы 1, нам надо воспользоваться для сумм (27) леммой 5 (см. приложение). Для этого необходимо выразить потенциалы $W(A)$ гамильтониана границы через $W(V \mid \vec{\partial})$, а потом найти оценку типа неравенств формулы (75) из приложения.

Для гамильтониана границы гамильтониан произвольного набора контуров границы непосредственно не определен (его задание нужно для введения функции Урселла, см. п. 2 приложения). Напомним необходимое определение раздела I.3. Для каждой границы $\vec{\partial}$ и набора контуров $\vec{\partial}_{1} \subset \vec{\partial}$ введем минимальное разбиение множества $\overrightarrow{\boldsymbol{\partial}}_{1}$ такое, что каждый элемент этого разбиения $\left[\overrightarrow{\boldsymbol{\partial}}_{1}\right]_{\vec{\partial}}$ уже является границей. Гамильтониан множества контуров $\vec{\partial}_{1}$ по определению равен

$$
H^{\vec{\partial}}\left(\vec{\partial}_{1}\right):=\sum_{\vec{\partial}_{2} \in\left[\vec{\partial}_{1}\right]_{\vec{\partial}}} H\left(\vec{\partial}_{2}\right)
$$

Следовательно, определены многочастичные потенциалы гамильтониана $H(\vec{\partial})$ и функции Урселла $\varphi([\vec{\partial}])$. Для каждого набора контуров $\vec{\partial}_{1} \subset \vec{\partial}$ определим также $\overrightarrow{\boldsymbol{\partial}}^{\mathrm{min}}\left(\overrightarrow{\boldsymbol{\partial}}_{1}\right) \in \gamma(\overrightarrow{\boldsymbol{\partial}})$ как минимальную границу, содержащую контуры из $\overrightarrow{\boldsymbol{\partial}}_{1}$. Ясно, что $\min ^{0} \vec{\partial}^{\text {min }}\left(\vec{\partial}_{1}\right)=\min ^{0} \vec{\partial}_{1}$.

Введем множество $R_{\gamma}(\vec{\partial})$ согласованных разбиений границы $\vec{\partial}$ (см. также раздел I.3). Каждый элемент этого множества $\overrightarrow{\mathbf{A}}$ состоит из набора границ: $\overrightarrow{\mathbf{A}}=$ $\left\{\overrightarrow{\boldsymbol{\partial}}_{1}, \ldots, \overrightarrow{\boldsymbol{\partial}}_{k}\right\}, \overrightarrow{\boldsymbol{\partial}}_{i} \in \gamma(\overrightarrow{\boldsymbol{\partial}}), \overrightarrow{\boldsymbol{\partial}}(\overrightarrow{\mathbf{A}}):=\cup \overrightarrow{\boldsymbol{\partial}}_{i}=\overrightarrow{\boldsymbol{\partial}}$. Мы можем ввести множество $\min ^{0} \overrightarrow{\mathbf{A}}$ как те границы $\vec{\partial}_{i} \in \overrightarrow{\mathbf{A}}$, носители которых лежат в некоторой связной компоненте $\mathbb{R}^{\nu} \backslash V_{\vec{\partial}(\overrightarrow{\mathbf{A}}) \backslash \overrightarrow{\boldsymbol{\partial}}_{1}}$

Лемма 1. Пусть $\overrightarrow{\mathbf{A}}=\left\{\overrightarrow{\boldsymbol{\partial}}_{1}, \ldots, \vec{\partial}_{n}\right\}, \vec{\partial}_{i} \in \gamma(\vec{\partial}), \vec{\partial}_{i} \cap \vec{\partial}_{j}=\varnothing \quad(i \neq j), \vec{\partial}(\overrightarrow{\mathbf{A}}) \subset \vec{\partial}$ $\operatorname{Ecлu} \overrightarrow{\boldsymbol{\partial}}(\overrightarrow{\mathbf{A}}) \notin \gamma(\overrightarrow{\boldsymbol{\partial}}), m o W^{\overrightarrow{\boldsymbol{\partial}}}(\overrightarrow{\mathbf{A}})=0 . \operatorname{Ec} л u \overrightarrow{\boldsymbol{\partial}}(\overrightarrow{\mathbf{A}}) \in \gamma(\overrightarrow{\boldsymbol{\partial}}), m o$

$$
W^{\overrightarrow{\boldsymbol{\partial}}}(\overrightarrow{\mathbf{A}})=W^{\overrightarrow{\boldsymbol{\partial}}(\overrightarrow{\mathbf{A}})}(\overrightarrow{\mathbf{A}})=W(\overrightarrow{\mathbf{A}})=W\left(\min ^{0} \overrightarrow{\mathbf{A}} \mid \overrightarrow{\mathbf{A}} \backslash \min ^{0} \overrightarrow{\mathbf{A}}\right) .
$$

Эти утверждения нетрудно получить из определений (38) и (68).

Пусть $\overrightarrow{\mathbf{A}}=\left\{\overrightarrow{\boldsymbol{\partial}}_{1},\left[\overrightarrow{\boldsymbol{\partial}}_{2}\right]_{\vec{\partial}}\right\}, \overrightarrow{\boldsymbol{\partial}}_{1} \in \gamma(\overrightarrow{\boldsymbol{\partial}}), \overrightarrow{\boldsymbol{\partial}}_{1} \cup \overrightarrow{\boldsymbol{\partial}}_{2} \in \gamma(\overrightarrow{\boldsymbol{\partial}})$ и $\overrightarrow{\boldsymbol{\partial}}_{1} \in \min ^{0} \overrightarrow{\mathbf{A}}$, следовательно, $\vec{\partial}_{2} \in \gamma(\vec{\partial})$. Тогда контуры из $\vec{\partial}_{1}$ можно пронумеровать так, что

$$
\begin{aligned}
\overrightarrow{\boldsymbol{\partial}}_{1} & =\left\{\overrightarrow{\boldsymbol{\Gamma}}_{1}, \ldots, \overrightarrow{\boldsymbol{\Gamma}}_{\left|\overrightarrow{\boldsymbol{\partial}}_{1}\right|}\right\}, \quad \overrightarrow{\boldsymbol{\partial}}_{2} \cup \overrightarrow{\boldsymbol{\partial}}_{1}\left(\overrightarrow{\boldsymbol{\Gamma}}_{i}\right) \in \gamma(\overrightarrow{\boldsymbol{\partial}}), \\
\overrightarrow{\boldsymbol{\partial}}_{1}\left(\overrightarrow{\boldsymbol{\Gamma}}_{i}\right) & :=\prod_{j \geqslant i+1} \overrightarrow{\boldsymbol{\Gamma}}_{j}, \quad \overrightarrow{\boldsymbol{\Gamma}}_{i} \in \min ^{0}\left(\overrightarrow{\boldsymbol{\Gamma}}_{i} \cup \overrightarrow{\boldsymbol{\partial}}_{2} \cup \overrightarrow{\boldsymbol{\partial}}_{1}\left(\overrightarrow{\boldsymbol{\Gamma}}_{i}\right)\right)
\end{aligned}
$$

Если $\overrightarrow{\mathbf{A}} \in R_{\gamma}(\overrightarrow{\boldsymbol{\partial}})$, тогда предыдущее построение можно провести для каждой границы $\vec{\partial}_{1} \in \min ^{0} \overrightarrow{\mathbf{A}}$.

Лемма 2. Имеют место следующие утверждения.

1. Если $\overrightarrow{\mathbf{A}} \in R_{\gamma}[\vec{\partial}]$, то в каждом из множеств $\vec{\partial}_{1} \in \overrightarrow{\mathbf{A}}$ нумерацию контуров можно выбрать такой, что при $\overrightarrow{\boldsymbol{\partial}}^{\prime} \stackrel{*}{\cap} \min ^{0} \overrightarrow{\mathbf{A}} \neq \varnothing\left(\right.$ m.e. $\left|\overrightarrow{\boldsymbol{\partial}}^{\prime}\right|=\left|\min ^{0} \overrightarrow{\mathbf{A}}\right| \neq 0$, 
$\left|\vec{\partial}^{\prime} \cap \vec{\partial}_{1}\right|=1$ для любой $\left.\vec{\partial}_{1} \in \min ^{0} \overrightarrow{\mathbf{A}}\right)$ въполненъ

$$
\begin{gathered}
\vec{\partial}^{\prime}=\min ^{0} \vec{\partial}^{\prime} \subset \min ^{0}\left(\vec{\partial}^{\prime} \cup \vec{\partial}_{\overrightarrow{\mathbf{A}}}\left(\vec{\partial}^{\prime}\right)\right), \\
\vec{\partial}_{\overrightarrow{\mathbf{A}}}\left(\vec{\partial}^{\prime}\right)=\vec{\partial}\left(\overrightarrow{\mathbf{A}} \backslash \min ^{0} \overrightarrow{\mathbf{A}}\right) \bigcup_{\overrightarrow{\boldsymbol{\Gamma}} \in \vec{\partial}^{\prime}} \vec{\partial}_{1}(\overrightarrow{\boldsymbol{\Gamma}}) \in \gamma(\vec{\partial})
\end{gathered}
$$

$\left(\overrightarrow{\boldsymbol{\partial}}_{1}(\overrightarrow{\boldsymbol{\Gamma}})\right.$ определено перед леммой $)$ u

$$
W\left(\min ^{0} \overrightarrow{\mathbf{A}} \mid \overrightarrow{\boldsymbol{\partial}}\left(\overrightarrow{\mathbf{A}} \backslash \min ^{0} \overrightarrow{\mathbf{A}}\right)\right)=\sum_{\vec{\partial}^{\prime} \cap \min ^{0} \overrightarrow{\mathbf{A}} \neq \varnothing} W\left(\left[\overrightarrow{\boldsymbol{\partial}}^{\prime}\right] \mid \vec{\partial}_{\overrightarrow{\mathbf{A}}}\left(\vec{\partial}^{\prime}\right)\right) .
$$

2. Пусть $\vec{\partial}_{1} \cup \vec{\partial}_{2} \in \gamma(\vec{\partial}), \vec{\partial}_{1}=\min ^{0} \vec{\partial}_{1} \subset \min ^{0}\left(\vec{\partial}_{1} \cup \vec{\partial}_{2}\right),\left|\vec{\partial}_{1}\right| \geqslant 2$, тогда

$$
\begin{aligned}
& W\left(\left[\overrightarrow{\boldsymbol{\partial}}_{1}\right] \mid \overrightarrow{\boldsymbol{\partial}}_{2}\right)=\sum_{V \cap \overrightarrow{\boldsymbol{\partial}}_{1} \neq \varnothing} \sum_{\overrightarrow{\boldsymbol{\partial}}_{3} \subset \overrightarrow{\boldsymbol{\partial}}_{1}}(-1)^{\left|\overrightarrow{\boldsymbol{\partial}}_{3}\right|} W\left(V \mid \overrightarrow{\boldsymbol{\partial}}_{2} \cup \overrightarrow{\boldsymbol{\partial}}_{3}\right)= \\
& =\sum_{V \stackrel{*}{\cap} \overrightarrow{\boldsymbol{\partial}}_{1} \neq \varnothing} \sum_{\overrightarrow{\boldsymbol{\partial}}_{3} \subset \overrightarrow{\boldsymbol{\partial}}_{1}}(-1)^{\left|\overrightarrow{\boldsymbol{\partial}}_{3}\right|} W_{1}\left(V \mid \overrightarrow{\boldsymbol{\partial}}_{2} \cup \overrightarrow{\boldsymbol{\partial}}_{3}\right)
\end{aligned}
$$

и справедливо неравенство

$$
|W(\overrightarrow{\mathbf{A}})| \leqslant \sum_{\vec{\partial}^{\prime} \cap \min ^{0} \overrightarrow{\mathbf{A}} \neq \varnothing} h(\overrightarrow{\boldsymbol{\Gamma}}) \overline{W_{1}}\left(\overrightarrow{\boldsymbol{\partial}}^{\prime}\right), \quad \overline{W_{1}}\left(\overrightarrow{\boldsymbol{\partial}}^{\prime}\right):=\sum_{V \stackrel{*}{\cap}^{\prime} \neq \varnothing} \bar{W}(V),
$$

где для каждого $\overrightarrow{\boldsymbol{\partial}}^{\prime}$ в (43) контур $\overrightarrow{\boldsymbol{\Gamma}}$ из $\vec{\partial}^{\prime}$ произволен, $\bar{W}(V)$ определено в (10). $V \stackrel{*}{\cap} \overrightarrow{\boldsymbol{\partial}}^{\prime} \neq \varnothing$ означает, что $V \cap V(\overrightarrow{\boldsymbol{\Gamma}}) \neq \varnothing$ для всех контуров, кроме внешнего; если $\vec{\partial}^{\prime}$ содержит внешний контур $\overrightarrow{\boldsymbol{\Gamma}}$, то $V \cap\left(R^{\nu} \backslash V(\overrightarrow{\boldsymbol{\Gamma}})\right) \neq \varnothing$.

Выбирая в каждом $\vec{\partial}_{i} \in \min ^{0} \overrightarrow{\mathbf{A}}$ последовательность контуров в соответствии с указанной перед леммой нумерацией, получаем (40), а затем, используя (39), получаем (41). Второе утверждение леммы вытекает из (39), (8) и условия локальности взаимодействия. Неравенство (43) есть прямое следствие формул (39), (41), (42) и (10). Для дальнейших рассуждений существенно, что из условия локальности вытекает, что $W_{1}(V \mid \overrightarrow{\boldsymbol{\partial}})$ и соответствующие слагаемые в (42) равны нулю, если $V$ целиком лежит в одной из компонент $\mathbb{R}^{\nu} \backslash V_{\vec{\partial}}$.

Перейдем непосредственно к доказательству теоремы 3. Рассмотрим ситуацию, когда $h(\overrightarrow{\boldsymbol{\Gamma}})=1$, и убедимся, что в этом случае верно неравенство $B(\overrightarrow{\boldsymbol{\Gamma}}) \leqslant r(\mu)|\overrightarrow{\boldsymbol{\Gamma}}|$ (см. (9)), где $r(\mu):=C_{4} \bar{W}_{2 x}(0, \mu)$. Чтобы получить нужное неравенство, рассмотрим разность $H_{1}(\overrightarrow{\boldsymbol{\partial}})-H_{1}(\overrightarrow{\boldsymbol{\partial}} \backslash \overrightarrow{\boldsymbol{\Gamma}})$, представив ее в виде $\left(q=k_{\mathrm{ex}}(\overrightarrow{\boldsymbol{\Gamma}}), V_{\mathrm{ex}}(\overrightarrow{\boldsymbol{\Gamma}})=R^{\nu} \backslash V(\overrightarrow{\boldsymbol{\Gamma}})\right.$, $\left.k_{i}=k_{i}(\overrightarrow{\boldsymbol{\Gamma}})\right)$

$$
\begin{aligned}
H_{1}(\overrightarrow{\boldsymbol{\partial}})-H_{1}(\overrightarrow{\boldsymbol{\partial}} \backslash \overrightarrow{\boldsymbol{\Gamma}})=\sum_{V} & {\left[W(V \mid \overrightarrow{\boldsymbol{\partial}})-\sum_{i \in l(\overrightarrow{\boldsymbol{\Gamma}})} W\left(V \mid k_{i}\right)+\right.} \\
+ & \left.\frac{\left|V \cap V_{\mathrm{ex}}(\overrightarrow{\boldsymbol{\Gamma}})\right|}{|V|} W(V \mid q)-W(V \mid \overrightarrow{\boldsymbol{\partial}} \backslash \overrightarrow{\boldsymbol{\Gamma}})\right] .
\end{aligned}
$$


Из условия локальности взаимодействия вытекает также, что выражение в квадратных скобках в предыдущей формуле равно нулю, если $V$ целиком лежит в одной из связных компонент $R^{\nu} \backslash V_{\overrightarrow{\boldsymbol{\Gamma}}}$. Следовательно, для отличных от нуля слагаемых найдутся либо $x \in V_{\overrightarrow{\boldsymbol{\Gamma}}}$, либо пара точек в $V$, для которых “прямая", их соединяющая, пересекает $\partial V_{\overrightarrow{\boldsymbol{\Gamma}}}$. Используя геометрические свойства границы (условие 1) и рассуждения леммы 5.3.7 из [23], находим, что

$$
\left|H_{1}(\overrightarrow{\boldsymbol{\partial}})-H_{1}(\overrightarrow{\boldsymbol{\partial}} \backslash \overrightarrow{\boldsymbol{\Gamma}})\right| \leqslant \bar{W}_{2 x}(0, \mu)\left(\left|\partial V_{\overrightarrow{\boldsymbol{\Gamma}}}\right|+\left|V_{\overrightarrow{\boldsymbol{\Gamma}}}\right|\right) \leqslant \bar{W}_{2 x}(0, \mu)|\overrightarrow{\boldsymbol{\Gamma}}| .
$$

Докажем неравенство (32). Используя неравенство п. 1 леммы 5 приложения и неравенство (43), имеем

$$
\left|z_{0}^{\vec{\partial}} \varphi([\vec{\partial}])\right| \leqslant \sum_{\left\{\eta_{\vec{\Gamma}}(\vec{\partial})\right\}} z^{\vec{\partial}} \prod_{\overrightarrow{\boldsymbol{\partial}}_{1} \in \pi(\eta)} h\left(\overrightarrow{\boldsymbol{\Gamma}}_{1}\right) \bar{W}\left(\overrightarrow{\boldsymbol{\partial}}_{1}\right) \leqslant \sum_{\left\{\eta_{\overrightarrow{\boldsymbol{\Gamma}}}(\overrightarrow{\boldsymbol{\partial}})\right\}}(z h)^{\overrightarrow{\boldsymbol{\partial}}} \prod_{\overrightarrow{\boldsymbol{\partial}}_{1} \in \pi(\eta)} \bar{W}\left(\overrightarrow{\boldsymbol{\partial}}_{1}\right),
$$

где $\bar{W}\left(\overrightarrow{\boldsymbol{\partial}}_{1}\right):=\bar{W}_{1}\left(\min ^{0} \overrightarrow{\boldsymbol{\partial}}_{1}\right)$. При переходе к последнему выражению каждый контур $\overrightarrow{\boldsymbol{\Gamma}}_{1} \in \min ^{0} \overrightarrow{\boldsymbol{\partial}}_{1}$ выбирался наиболее удаленным от корня “дерева" $\eta_{\overrightarrow{\boldsymbol{\Gamma}}}(\overrightarrow{\boldsymbol{\partial}})$.

Следующий шаг получения необходимых нам оценок состоит в суммировании по всем границам $\vec{\partial}$, для которых $V_{\vec{\partial}}=$ const и $q=k_{\text {ex }}(\vec{\Gamma})=$ const. Ниже в этом разделе нам удобно положить $\bar{\partial}=V_{\vec{\partial}}, \bar{\Gamma}=V_{\overrightarrow{\boldsymbol{\Gamma}}}, \bar{W}(\bar{\partial})=\max _{\vec{\partial}}: \bar{\partial}=V_{\vec{\partial}} \bar{W}(\vec{\partial})$. Введем также функционал

$$
\bar{z}(\bar{\Gamma}):=\max _{q} \sum_{\substack{\overrightarrow{\boldsymbol{\Gamma}}: \bar{\Gamma}=V_{\vec{\Gamma}} \\ V_{q}^{\prime}(\overrightarrow{\boldsymbol{\Gamma}}) \neq \varnothing}} h(\overrightarrow{\boldsymbol{\Gamma}}) z(\overrightarrow{\boldsymbol{\Gamma}}) .
$$

Суммирование по $\vec{\partial}_{1}$ в (45) при условиях $V_{\vec{\partial}}=$ const и $q=k_{\mathrm{ex}}(\overrightarrow{\boldsymbol{\Gamma}})=$ const начнем с контуров $\overrightarrow{\boldsymbol{\Gamma}}_{1}$, наиболее удаленных от "точки" $\overrightarrow{\boldsymbol{\partial}}$-графа (см. раздел I.1, где дано определение $\vec{\partial}$-графа), для которой $V_{q}^{\prime}(\overrightarrow{\boldsymbol{\Gamma}}) \cap V_{i}(\overrightarrow{\boldsymbol{\Gamma}}) \neq \varnothing$. Тип фазы и соответствующий объем $V_{i}\left(\overrightarrow{\boldsymbol{\Gamma}}_{1}\right)$ “точки” $\overrightarrow{\boldsymbol{\partial}}$-графа, посредством которой контур $\overrightarrow{\boldsymbol{\Gamma}}_{1}$ "присоединен” к $\vec{\partial}$-графу, фиксированы, и тем самым результат суммирования по $\overrightarrow{\boldsymbol{\Gamma}}_{1}$ при фиксированных $\overrightarrow{\boldsymbol{\partial}} \backslash \overrightarrow{\boldsymbol{\Gamma}}_{1}$ и $V_{\overrightarrow{\boldsymbol{\Gamma}}_{1}}$ не превосходит $\bar{z}(\bar{\Gamma})$. При помощи индукции отсюда выводим неравенство

$$
\max _{q} \sum_{\substack{\overrightarrow{\boldsymbol{\partial}} \ni \overrightarrow{\boldsymbol{\Gamma}}: V_{q}^{\prime}(\overrightarrow{\boldsymbol{\Gamma}}) \neq \varnothing, V_{\vec{\partial}}=\text { const }}}\left|z_{0}^{\overrightarrow{\boldsymbol{\partial}}} \varphi([\overrightarrow{\boldsymbol{\partial}}])\right| \leqslant \sum_{\left\{\eta_{\bar{\Gamma}(\bar{\partial})}\right\}} \bar{z}^{\bar{\partial}} \prod_{\bar{\partial}_{1} \in \pi(\eta)} \bar{W}\left(\bar{\partial}_{1}\right)
$$

Таким образом, правая часть (47) зависит лишь от геометрической границы $\bar{\partial}$, а вся зависимость от фаз “скрыта" в $\bar{z}(\bar{\Gamma})$ (см. (46)).

Из п. 2 леммы 5 вытекает, что правая часть $(47)$ не превосходит $\bar{\omega}(\bar{\Gamma})$, если

$$
\bar{z}(\bar{\Gamma}) \exp \left\{\sum_{\bar{\partial}} \bar{W}(\bar{\Gamma} \cup \bar{\partial}) \bar{\omega}^{\partial}\right\} \leqslant \bar{\omega}(\bar{\Gamma}) .
$$

Убедимся, что

$$
\sum_{\bar{\partial}} \bar{W}(\bar{\Gamma} \cup \bar{\partial}) \bar{\omega}^{\bar{\partial}} \leqslant C_{2}|\bar{\Gamma}| \tilde{a} \bar{W}_{2 x}(\tilde{a}, \mu)
$$


где $\bar{W}_{2 x}$ определено в условии 3 теоремы 1 , а

$$
\tilde{a}=\sum_{\bar{\Gamma}: 0 \in V(\bar{\Gamma})} \bar{\omega}(\bar{\Gamma}) \exp \left\{\sum_{\bar{\Gamma}: 0 \in V(\bar{\Gamma})} \bar{\omega}(\bar{\Gamma})\right\} .
$$

Из формул (43) и определения $\bar{W}(\partial)$ находим, что

$$
\sum_{\bar{\partial}} \bar{W}(\bar{\Gamma} \cup \bar{\partial}) \bar{\omega}^{\bar{\partial}} \leqslant \sum_{\substack{\bar{\partial} \ni \bar{\Gamma}, V: V \stackrel{*}{*} \min ^{0} \bar{\partial} \neq \varnothing}} \bar{W}(V) \bar{\omega}^{\bar{\partial} \backslash \bar{\Gamma}},
$$

где условия суммирования в правой части определены в конце леммы 2. В каждом из множеств $V \cap V\left(\bar{\Gamma}_{1}\right)$ или $V \cap V_{\text {ex }}\left(\bar{\Gamma}_{1}\right), \bar{\Gamma}_{1} \in \min ^{0} \bar{\partial}$ выделим лексикографически минимальную точку; совокупность таких точек обозначим $V_{1}$. Итак, по построению $V_{1} \stackrel{*}{\cap} \bar{\partial} \neq \varnothing,\left|V_{1}\right| \geqslant 2$, кроме того, в $V_{1}$ найдется пара точек $x_{1}, x_{2}$ таких, что "отрезок", их соединяющий, пересекает $\partial V(\bar{\Gamma})$. Аналогично доказательству (44) нетрудно получить следующее. Если $\bar{\Gamma}$ - внешний контур $\bar{\partial}$, то соответствующая сумма в правой части (50) не превосходит $\tilde{a}(1+\tilde{a})^{-1} \bar{W}_{2 x}(\tilde{a}, \mu)\left|\partial V_{\bar{\Gamma}} \backslash \partial V(\bar{\Gamma})\right|$. При $\bar{\Gamma} \in \min ^{0} \bar{\partial}$ сумма в правой части $(50)$ не превосходит $\tilde{a}(1+\tilde{a})^{-1} \bar{W}_{2 x}(\tilde{a}, \mu)|\partial V(\bar{\Gamma})|$. Если $\bar{\Gamma} \notin \min ^{0} \bar{\partial}$, то оценка правой части (50) имеет вид $\tilde{a}^{2}(1+\tilde{a})^{-2} \bar{W}_{2 x}(\tilde{a}, \mu)\left|\partial V_{\bar{\Gamma}}\right|$. В каждом случае мы проводим сначала суммирование по величинам $\bar{\omega}^{\bar{\lambda}} \backslash \bar{\Gamma}$, что дает результат, не превосходящий $\tilde{a}^{\left|V_{1}\right|-1}$ или $\tilde{a}^{\left|V_{1}\right|-2}$, затем суммирование по $V_{1}$ в $V$, что дает результат не более $(1+\tilde{a})^{|V|-1}$ или $(1+\tilde{a})^{|V|-2}$, потом суммирование $(1+\tilde{a})^{|V|-1} \bar{W}(V)$ по $V$ при фиксированных $x_{1}, x_{2}$, а затем по $x_{1}, x_{2}$, что и дает (49). "Сокращая" число переменных в (48) (см. последнее утверждение леммы 5), положим $\bar{\omega}(\bar{\Gamma})=\bar{z}(\bar{\Gamma}) e^{\lambda|\bar{\Gamma}|}$, тогда для справедливости (48) достаточно, чтобы было выполнено неравенство $\lambda \geqslant C_{2} \tilde{a} \bar{W}_{2 x}(\tilde{a}, \mu)$, где в правой части $(49) \bar{\omega}(\bar{\Gamma})=\bar{z}(\bar{\Gamma}) e^{\lambda|\bar{\Gamma}|}$. Таким образом, неравенства (32) доказаны, и нам осталось доказать (33).

В правой части (33) используем неравенство (45), в котором $\bar{\Gamma}=\bar{\Gamma}^{\prime}=V_{\bar{\Gamma}^{\prime}}$. Так как каждому слагаемому в полученной оценке соответствует граф типа “дерева" (вклад графа в сумму равен $\left.z^{\bar{\partial}} \prod \bar{W}\left(\bar{\partial}_{j}\right)\right)$, найдется единственная последовательность потенциалов $\bar{W}\left(\bar{\partial}_{i}\right)$ и контуров $\bar{\Gamma}_{i}$ таких, что $\bar{\Gamma}^{\prime} \equiv \bar{\Gamma}_{1} \in \bar{\partial}_{1}, \bar{\Gamma}^{\prime \prime} \equiv \bar{\Gamma}_{n} \in \bar{\partial}_{n}, \bar{\partial}_{i} \cap \bar{\partial}_{i+1}=\bar{\Gamma}_{i}$. Выберем из последовательности контуров $\bar{\Gamma}_{i}$ подпоследовательность следующим образом: найдем два контура $\bar{\Gamma}_{i_{1}} \equiv \bar{\Gamma}_{1}^{2}$ и $\bar{\Gamma}_{i_{1}+1} \equiv \bar{\Gamma}_{2}^{1}$ таких, что $\bar{\Gamma}_{j} \subset V\left(\bar{\Gamma}_{1}\right), j=1, \ldots, i_{1}$, и $V\left(\bar{\Gamma}_{i_{1}+1}\right) \cap V\left(\bar{\Gamma}_{1}\right)=\varnothing, \bar{\Gamma}_{1}^{1}=\bar{\Gamma}^{1}=\bar{\Gamma}^{\prime}$; найдем следующую пару контуров $\bar{\Gamma}_{i_{2}} \equiv \bar{\Gamma}_{2}^{2}$ и $\bar{\Gamma}_{i_{2}+1} \equiv \bar{\Gamma}_{3}^{1}$ таких, что $\bar{\Gamma}_{j} \subset V\left(\bar{\Gamma}_{2}^{1}\right), j=i_{1}+1, \ldots, i_{2}$, и $V\left(\bar{\Gamma}_{i_{2}+1}\right) \cap V\left(\bar{\Gamma}_{2}^{1}\right)=\varnothing$ и т.д., пока контур $\bar{\Gamma}_{k}^{1}$ не окажется равным или лежащим внутри $\bar{\Gamma}^{\prime \prime}$. Таким способом определена последовательность контуров $\bar{\Gamma}_{j-1}^{2}, \bar{\Gamma}_{j}^{1}, j=2, \ldots, k$, для которой во-первых, $\bar{\Gamma}_{j}^{2} \subset V\left(\bar{\Gamma}_{j}^{1}\right)$ или $\bar{\Gamma}_{j}^{2}=\bar{\Gamma}_{j}^{1}$; во-вторых, $V\left(\bar{\Gamma}_{j}^{1}\right) \cap V\left(\bar{\Gamma}_{j+1}^{1}\right)=\varnothing ;$ в-третьих, $\bar{\Gamma}_{j}^{2} \in \bar{\partial}_{i_{j}}, \bar{\Gamma}_{j+1}^{1} \in \bar{\partial}_{i_{j}}$, где $i_{j}$ - возрастающая подпоследовательность номеров $1, \ldots, n ;$ в-четвертых, $\bar{\Gamma}_{j}^{1} \equiv \bar{\Gamma}^{\prime}, \bar{\Gamma}_{k}^{1} \subset V\left(\bar{\Gamma}^{\prime \prime}\right)$ или $\bar{\Gamma}_{k}^{1}=V\left(\bar{\Gamma}^{\prime \prime}\right)$. Представим каждый из потенциалов $\bar{W}\left(\bar{\partial}_{i_{j}}\right)$ в виде суммы по $V_{j}, j=1, \ldots, k-1$, и выделим в каждом $V_{j}$ пару точек $x_{2 j-1} \in V\left(\bar{\Gamma}_{j}^{2}\right) \subset V\left(\bar{\Gamma}_{j}^{1}\right)$ и $x_{2 j} \in V\left(\bar{\Gamma}_{j+1}^{1}\right)$. Увеличим $d\left(\bar{\Gamma}^{\prime}, \bar{\Gamma}^{\prime \prime}\right)$ до $\left|x_{1}-x_{2 k-2}\right|$ и проведем суммирование по всем контурам, кроме $\bigcup_{j} \bar{\partial}_{i_{j}}$, в результате будем иметь

5 Теоретическая и математическая физика, т. 153, № 1, 2007 г. 
не более $\bar{\omega}(\bar{\Gamma})$ для каждого контура из этого множества; затем суммируем по $\bar{\partial}_{i_{j}}, V_{j}$, для которых фиксированы точки $x_{2 j-1}, x_{2 j}$ и по контурам $\bar{\Gamma}_{j}^{1}$, действуя при этом так же, как при доказательстве (32). Таким образом, оценкой левой части (33) является

$$
\sum_{k \geqslant 2} \sum_{x_{1}, \ldots, x_{2 k-2}}\left|x_{1}-x_{2 k-2}\right| \sum \prod_{i=1}^{k} \bar{\omega}(\bar{\Gamma}) \prod_{i=1}^{k-1} \bar{W}_{2}\left(x_{2 i-1}-x_{2 i} ; \tilde{a}, \mu\right),
$$

где в последней сумме условия суммирования следующие: $\bar{\Gamma}_{1}, \ldots, \bar{\Gamma}_{k}$ таковы, что $x_{1} \in V\left(\bar{\Gamma}_{1}\right), x_{2 k-2} \in V\left(\bar{\Gamma}_{k}\right), x_{2 i} \cup x_{2 i+1} \subset V\left(\bar{\Gamma}_{i}\right)$ для любого $i$. После использования неравенства $\left|x_{1}-x_{2 k-2}\right| \leqslant \sum\left|x_{i}-x_{i+1}\right|$ и определений (30), (31), получим (33). Наши оценки верны и после взятия формальных производных в соответствующих неравенствах. Теорема 3, а вместе с ней и теорема 1 доказаны.

\section{4. РАСШИРЕНИЕ ОБЛАСТИ ФАЗОВЫХ ПЕРЕХОДОВ ПЕРВОГО РОДА}

Построение, дающее расширение области фазовых переходов первого рода, основано на том, что сдвиг внешних полей в (1) можно записать как соответствующий сдвиг контурного функционала $H_{0}(\overrightarrow{\boldsymbol{\Gamma}})$. При чисто мнимом сдвиге $H_{0}(\overrightarrow{\boldsymbol{\Gamma}})$ оценки теоремы 1 остаются верными! Следовательно, наше рассмотрение оказывается верным в более широкой области. Если раньше мы могли утверждать, что теорема 1 верна лишь в вещественной части областей $\Im, \mathcal{M}$, а также в допустимой комплексной окрестности областей $\Im_{\mathbb{R}}, \mathcal{M}_{\mathbb{R}}$, то теперь мы можем расширить область фазовых переходов первого рода до областей $\Im, \mathcal{M}$.

Пусть задан набор вещественных чисел $\alpha_{q}, q \in \Phi$. Введем функционалы

$$
\begin{gathered}
H_{0}(\overrightarrow{\boldsymbol{\Gamma}}, \overrightarrow{\boldsymbol{\alpha}}):=H_{0}(\overrightarrow{\boldsymbol{\Gamma}})+i\left[\alpha_{k_{\mathrm{ex}}(\overrightarrow{\boldsymbol{\Gamma}})}|V(\overrightarrow{\boldsymbol{\Gamma}})|-\sum_{j \in l(\overrightarrow{\boldsymbol{\Gamma}})} \alpha_{k_{j}(\overrightarrow{\boldsymbol{\Gamma}})}\left|V_{j}(\overrightarrow{\boldsymbol{\Gamma}})\right|\right], \\
H(\overrightarrow{\boldsymbol{\partial}}, \overrightarrow{\boldsymbol{\alpha}}):=\sum_{\overrightarrow{\boldsymbol{\Gamma}} \in \overrightarrow{\boldsymbol{\partial}}} H_{0}(\overrightarrow{\boldsymbol{\Gamma}}, \overrightarrow{\boldsymbol{\alpha}})+H_{1}(\overrightarrow{\boldsymbol{\partial}}) .
\end{gathered}
$$

Лемма 3. Статистические суммы (1) многофазных контурных моделей fc(field-contour)-инвариантны, т.е. для них справедливы тождества

$$
\Xi_{q}(V, H, \overrightarrow{\mathbf{h}})=e^{i \alpha_{q}|V|} \Xi_{q}(V, H(\overrightarrow{\boldsymbol{\alpha}}), \overrightarrow{\mathbf{h}}-i \overrightarrow{\boldsymbol{\alpha}}) .
$$

Равенство (52) вытекает из "почти геометрического" тождества $\left(V_{\vec{\partial}} \subset V\right)$

$$
\begin{aligned}
& \alpha_{k_{\mathrm{ex}}(\overrightarrow{\boldsymbol{\partial}})}|V \backslash V(\overrightarrow{\boldsymbol{\partial}})|+\sum_{j \in l(\overrightarrow{\boldsymbol{\partial}})} \alpha_{k_{j}(\overrightarrow{\boldsymbol{\partial}})}\left|V_{j}(\overrightarrow{\boldsymbol{\partial}})\right|= \\
& =\alpha_{k_{\mathrm{ex}}(\overrightarrow{\boldsymbol{\partial}})}|V|-\sum_{\overrightarrow{\boldsymbol{\Gamma}} \in \overrightarrow{\boldsymbol{\partial}}}\left[\alpha_{k_{\mathrm{ex}}(\overrightarrow{\boldsymbol{\Gamma}})}|V(\overrightarrow{\boldsymbol{\Gamma}})|-\sum_{j \in l(\overrightarrow{\boldsymbol{\Gamma}})} \alpha_{k_{j}(\overrightarrow{\boldsymbol{\Gamma}})}\left|V_{j}(\overrightarrow{\boldsymbol{\Gamma}})\right|\right] .
\end{aligned}
$$

Пусть $\overrightarrow{\boldsymbol{\partial}}$ - граница, состоящая из внешних контуров. Тогда (53), очевидно, выполнено. Пусть $\overrightarrow{\boldsymbol{\partial}}, \overrightarrow{\boldsymbol{\partial}} \cup \overrightarrow{\boldsymbol{\partial}}_{1}$ - границы, $\min ^{0} \overrightarrow{\boldsymbol{\partial}}_{1}=\overrightarrow{\boldsymbol{\partial}}_{1}, V_{\overrightarrow{\boldsymbol{\partial}}_{1}} \subset V_{i}(\overrightarrow{\boldsymbol{\partial}})$ и пусть для $\overrightarrow{\boldsymbol{\partial}}$ тождество (53) выполнено. В этом случае предыдущее рассуждение надо повторить для области $V_{i}(\vec{\partial})$. 
ОПРЕдЕЛЕНИЕ 3 . Модель со статистическими суммами $\Xi_{q}(V, H(\overrightarrow{\boldsymbol{\alpha}}), \overrightarrow{\mathbf{h}}-i \overrightarrow{\boldsymbol{\alpha}})$ будем называть контурной $\alpha$-моделъю, соответствующей модели (1).

Так как $\alpha$-модель - это стандартная многофазная контурная модель (1), у которой лишь переопределены гамильтониан и внешние поля, к ней для каждого $\overrightarrow{\boldsymbol{\alpha}}$ применимы построения контурных уравнений и уравнения состояния.

Мы будем строить контурные уравнения и уравнения состояния двумя способами.

В первом случае в построениях раздела I.5 для статистических сумм $\alpha$-модели вектор давлений всегда вещественен и равен $\operatorname{Re} \overrightarrow{\mathbf{p}}$, а вектор $\overrightarrow{\boldsymbol{\alpha}}=\operatorname{Im} \overrightarrow{\mathbf{p}}$. В этом случае контурные уравнения и уравнения состояния мы рассматриваем на пространстве $\wp$, где

$$
\begin{gathered}
\wp:=\mathbb{C}^{\Phi} \times \mathcal{M}_{p}=\left\{(\overrightarrow{\mathbf{p}}, \mu): \overrightarrow{\mathbf{p}} \in \mathbb{C}^{\Phi}, \mu \in \mathcal{M}_{p}\right\}=\bigcup_{G \subset \Phi} \partial_{G \wp}, \\
\partial_{G \wp}:=\left\{(\overrightarrow{\mathbf{p}}, \mu) \in \wp:\left.\operatorname{Re} \overrightarrow{\mathbf{p}}\right|_{G}=\left.p_{\max , \mathbb{R}} \overrightarrow{\mathbf{1}}_{\mathbb{R}}\right|_{G},\left.\operatorname{Re} \overrightarrow{\mathbf{p}}\right|_{\Phi \backslash G}<\left.p_{\max , \mathbb{R}} \overrightarrow{\mathbf{1}}_{\mathbb{R}}\right|_{\Phi \backslash G}, \mu \in \mathcal{M}_{p}\right\},
\end{gathered}
$$

$\mathcal{M}_{p}$ определено в $(21)$ после замены $\max \operatorname{Re} h_{q}$ на $p_{\max , \mathbb{R}}$. Для простоты формулировок мы используем в определении ю величину (23), а не (11). Уравнение состояния $\overrightarrow{\mathbf{h}}(\overrightarrow{\mathbf{p}}, \mu)-i \operatorname{Im} \overrightarrow{\mathbf{p}}=\operatorname{Re} \overrightarrow{\mathbf{p}}-\overrightarrow{\mathbf{s}}_{\operatorname{Im} \overrightarrow{\mathbf{p}}}(\operatorname{Re} \overrightarrow{\mathbf{p}}, \mu)$ и контурные уравнения на ю запишем в виде

$$
\begin{gathered}
\overrightarrow{\mathbf{h}}(\overrightarrow{\mathbf{p}}, \mu)=\overrightarrow{\mathbf{p}}-\overrightarrow{\mathbf{s}}_{\operatorname{Im} \overrightarrow{\mathbf{p}}}(\operatorname{Re} \overrightarrow{\mathbf{p}}, \mu), \\
\overrightarrow{\mathcal{F}}=\overrightarrow{\boldsymbol{\Upsilon}}_{\operatorname{Im} \overrightarrow{\mathbf{p}}}(\overrightarrow{\mathcal{F}}, \operatorname{Re} \overrightarrow{\mathbf{p}}, \mu), \quad(\overrightarrow{\mathbf{p}}, \mu) \in \wp,
\end{gathered}
$$

где индекс у векторов $\overrightarrow{\mathbf{s}}, \overrightarrow{\boldsymbol{\Upsilon}}$ равен сдвигу в (52). Из теоремы 1 и формулы (52) вытекает, что давление в модели (1) равно $p_{\max , q}(\overrightarrow{\mathbf{h}}, \mu)=p_{\max , \mathbb{R}}(\overrightarrow{\mathbf{h}}, \mu)+i \operatorname{Im} p_{q}(\overrightarrow{\mathbf{h}}, \mu)$, $q \in G(\mu)$.

Во втором случае мы выбираем точку $\overrightarrow{\mathbf{p}}_{0} \in \partial_{G} \wp$. Далее, $\overrightarrow{\boldsymbol{\alpha}}:=\operatorname{Im} \overrightarrow{\mathbf{p}}_{0}$, вектор давлений в построении контурных уравнений и уравнения состояния равен $\overrightarrow{\mathbf{p}}-i \overrightarrow{\boldsymbol{\alpha}}$, он вещественен на компонентах из $G$, а сами эти компоненты равны между собой. Контурные уравнения и уравнение состояния мы строим на множестве

$$
\begin{aligned}
\partial_{G} \wp_{\operatorname{Im} \overrightarrow{\mathbf{p}}_{0}}^{\mathbb{C}:=} & \left\{(\overrightarrow{\mathbf{p}}, \mu): \overrightarrow{\mathbf{p}} \in \mathbb{C}^{\Phi},\left.\operatorname{Im} \overrightarrow{\mathbf{p}}\right|_{G}=\left.\operatorname{Im} \overrightarrow{\mathbf{p}}_{0}\right|_{G},\right. \\
& \left.\left.\operatorname{Re} \overrightarrow{\mathbf{p}}\right|_{G}=\left.p_{\max , \overrightarrow{\boldsymbol{\alpha}} \overrightarrow{\mathbf{1}}_{\mathbb{R}}}\right|_{G}, e^{\Delta p_{q}-i \alpha_{q}} \in S_{1}\left(a_{q}\right) \forall q \in \Phi \backslash G, \mu \in \mathcal{M}_{p}\right\} .
\end{aligned}
$$

Страт $(56)$ содержится в страте $\partial_{G} \wp$. Отображение $\overrightarrow{\mathbf{p}} \rightarrow \overrightarrow{\mathbf{h}}$ и соответствующие контурные уравнения запишем в виде

$$
\begin{gathered}
\overrightarrow{\mathbf{h}}_{\operatorname{Im} \overrightarrow{\mathbf{p}}_{0}}(\overrightarrow{\mathbf{p}}, \mu)=\overrightarrow{\mathbf{p}}-\overrightarrow{\mathbf{s}}_{\operatorname{Im} \overrightarrow{\mathbf{p}}_{0}}\left(\overrightarrow{\mathbf{p}}-i \operatorname{Im} \overrightarrow{\mathbf{p}}_{0}, \mu\right), \quad(\overrightarrow{\mathbf{p}}, \mu) \in \partial_{G} \wp_{\operatorname{Im} \overrightarrow{\mathbf{p}}_{0}}^{\mathbb{C}}, \\
\overrightarrow{\mathcal{F}}=\overrightarrow{\boldsymbol{\Upsilon}}_{\operatorname{Im} \overrightarrow{\mathbf{p}}_{0}}\left(\overrightarrow{\mathcal{F}}, \overrightarrow{\mathbf{p}}-i \operatorname{Im} \overrightarrow{\mathbf{p}}_{0}, \mu\right) .
\end{gathered}
$$

"Вещественная" часть страта $\partial_{G} \wp_{\overrightarrow{\boldsymbol{\alpha}}}^{\mathbb{C}}$ равна

$$
\partial_{G} \wp_{\overrightarrow{\boldsymbol{\alpha}}}^{\mathbb{C}, \mathbb{R}}:=\left\{(\overrightarrow{\mathbf{p}}, \mu) \in \partial_{G \wp_{\overrightarrow{\boldsymbol{\alpha}}}}^{\mathbb{C}}: \overrightarrow{\mathbf{p}}-\left.i \overrightarrow{\boldsymbol{\alpha}}\right|_{\Phi \backslash G} \in \mathbb{R}^{\Phi \backslash G}, e^{\Delta \overrightarrow{\mathbf{p}}-i \Delta \overrightarrow{\boldsymbol{\alpha}}}<\left.\overrightarrow{\mathbf{1}}_{\mathbb{R}}\right|_{\Phi \backslash G}, \mu \in \mathcal{M}_{p}\right\} .
$$

Введем также

$$
\partial_{G \wp^{\mathbb{C}}:=} \bigcup_{\overrightarrow{\boldsymbol{\alpha}} \in \mathbb{R}^{\Phi}:\left.\overrightarrow{\boldsymbol{\alpha}}\right|_{G}=\left.\alpha_{G} \overrightarrow{\mathbf{1}}\right|_{G}} \partial_{G} \wp_{\overrightarrow{\boldsymbol{\alpha}}}^{\mathbb{C}}, \quad \wp^{\mathbb{C}}:=\bigcup_{G \in \Phi} \partial_{G} \wp^{\mathbb{C}} .
$$


Так как все компоненты вектора $\overrightarrow{\mathbf{p}}$ с индексами из $G$ в страте $\partial_{G \wp^{\mathbb{C}}}$ равны как комплексные числа, обозначим давление $p_{\max , \mathbb{C}}$. Нетрудно видеть, что

$$
\partial_{G \wp^{\mathbb{C}}}:=\left\{(\overrightarrow{\mathbf{p}}, \mu): \overrightarrow{\mathbf{p}} \in \mathbb{C}^{\Phi},\left.\overrightarrow{\mathbf{p}}\right|_{G}=\left.p_{\max , \mathbb{C}} \overrightarrow{\mathbf{1}}_{\mathbb{C}}\right|_{G},\left|e^{\Delta p_{q}}\right|<1 \forall q \in \Phi \backslash G, \mu \in \mathcal{M}_{p}\right\} .
$$

Отметим, что все страты по переменным $\overrightarrow{\mathbf{p}}$ являются плоскими. Страт $\partial_{G \wp}$ имеет вещественную коразмерность $|G|-1$, страт $\partial_{G} \wp^{\mathbb{C}} \subset \partial_{G \wp}-$ комплексную коразмерность $|G|-1$. Ясно также, что эти страты отличаются лишь из-за возможности (невозможности) изменения мнимой части вектора давления.

Из (57) и (55) вытекает, что

$$
\overrightarrow{\mathbf{h}}_{\overrightarrow{\boldsymbol{\alpha}}}(\overrightarrow{\mathbf{p}}, \mu)=\overrightarrow{\mathbf{h}}(\overrightarrow{\mathbf{p}}, \mu), \quad(\overrightarrow{\mathbf{p}}, \mu) \in \partial_{G} \wp_{\overrightarrow{\boldsymbol{\alpha}}}^{\mathbb{C}, \mathbb{R}}, \quad \overrightarrow{\boldsymbol{\alpha}}=\operatorname{Im} \overrightarrow{\mathbf{p}}=\text { const } .
$$

Обратное отображение к (55), задающее давление, является функцией в области э:

$$
\overrightarrow{\mathbf{p}}=\overrightarrow{\mathbf{p}}(\overrightarrow{\mathbf{h}}, \mu) .
$$

Если внешние поля фаз нельзя считать не зависящими от параметров модели, то уравнение состояния определено на $\mathcal{M}$ и принимает вид

$$
\overrightarrow{\mathbf{p}}(\mu)=\overrightarrow{\mathbf{p}}(\overrightarrow{\mathbf{h}}(\mu), \mu) .
$$

Заметим, что отображение $\overrightarrow{\mathbf{h}}(\overrightarrow{\mathbf{p}}, \mu)$, вообще говоря, неаналитично, так как в нем мы “неаналитично разъединили" зависимость от вещественной и мнимой частей вектора давлений $\overrightarrow{\mathbf{p}}$. Отображение $\overrightarrow{\mathbf{h}}_{\overrightarrow{\boldsymbol{\alpha}}}(\overrightarrow{\mathbf{p}}, \mu),(\overrightarrow{\mathbf{p}}, \mu) \in \partial_{G} \wp_{\overrightarrow{\boldsymbol{\alpha}}}^{\mathbb{C}}$, напротив, аналитично для каждого $\overrightarrow{\boldsymbol{\alpha}}$ по $\overrightarrow{\mathbf{p}}$, что и позволяет "вернуть" аналитичность $p_{\max , q}(\overrightarrow{\mathbf{h}}, \mu)$ в каждом страте $\partial_{G} \Im^{\mathbb{C}}$. Действительно, для самого́ давления $p_{\max , q}(\overrightarrow{\mathbf{h}}, \mu)$ или $p_{\max , \mathbb{C}}(\overrightarrow{\mathbf{h}}, \mu)$ мы имеем определение на основании термодинамического предела. Контурные уравнения и теорема 1 гарантируют существование термодинамического предела этих двух величин и, так как предел единственен, их равенство. Таким образом, эти величины равны в пересечении своих областей определения, по крайней мере в комплексной окрестности в страте $\partial_{G} \Im^{\mathbb{C}}$ точки $(\overrightarrow{\mathbf{h}}, \mu)$. Сам страт $\partial_{G} \Im^{\mathbb{C}}$ является комплексным многообразием коразмерности $|G|-1$.

Нам осталось доказать аналитичность давления в гладких стратах $\partial_{G} \wp$ по части переменных. Возьмем точку в страте $\partial_{G} \wp$ (или соответствующую точку в $\partial_{G} \Im$ ) и убедимся, что давление является голоморфным в области $\left.\operatorname{Re} \Delta \overrightarrow{\mathbf{p}}\right|_{\Phi \backslash G}(\overrightarrow{\mathbf{h}}, \mu)<0$, т.е. в подмногообразии $\partial_{G} \Im$, определяемом уравнением $\left.\overrightarrow{\mathbf{p}}\right|_{G}(\overrightarrow{\mathbf{h}}, \mu)=$ const. Таким образом, в окрестности каждой точки в страте гладкого многообразия $\partial_{G} \Im$ (его вещественная коразмерность равна $|G|-1)$ мы выделяем комплексное подмногообразие комплексной размерности $|\Phi \backslash G|$ с помощью уравнения $\left.\overrightarrow{\mathbf{p}}\right|_{G}(\overrightarrow{\mathbf{h}}, \mu)=$ const. Чтобы убедиться, что давление $p_{q}(\overrightarrow{\mathbf{h}}, \mu), q \in G$, является голоморфным в области $\left.\operatorname{Re} \Delta \overrightarrow{\mathbf{p}}(\overrightarrow{\mathbf{h}}, \mu)\right|_{\Phi \backslash G}<0$, нам осталось воспользоваться рассуждениями предыдущего абзаца для давления каждой из устойчивых фаз, откуда и получим необходимое свойство давления.

Итак справедлива следующая теорема.

ТеОрема 4. Пусть выполнены условия 1-5 теоремы 1 при замене $H_{0}(\overrightarrow{\boldsymbol{\Gamma}})$ на $W^{\Lambda}(V \mid \overrightarrow{\boldsymbol{\partial}})$ и $H_{0}^{\Lambda}(\overrightarrow{\boldsymbol{\Gamma}})$, начиная с некоторого $\Lambda$ при $\Lambda \uparrow \mathbb{Z}^{\nu}$. Тогда области ю,, $\mathcal{M}$ 
(см. (37) или (21) при достаточно мальх $\left.\psi_{0}, \psi_{1}\right)$ являются областями фазовых переходов первого рода, в которых верны теоремы I.4-I.7 и, в частности, следующие утверждения.

1. Контурное уравнение (второе уравнение в (55)) имеет решения в области $\wp=\mathbb{C}^{\Phi} \times \mathcal{M}$, и в этой области определено непрерывное отображение $\overrightarrow{\mathbf{h}}(\overrightarrow{\mathbf{p}}, \mu)$.

2. Решение контурного уравнения и отображение $\overrightarrow{\mathbf{h}}(\overrightarrow{\mathbf{p}}, \mu)$ разлагаются в сходящиеся (равномерно в ю) ряды по степеням $\varphi_{\vec{\partial}}$, эти функиии $C^{\infty}$-гладки в каждом страте $\partial_{G \wp}$ и продолжаются до аналитических в области $\left.\operatorname{Re} \Delta \overrightarrow{\mathbf{p}}\right|_{\Phi \backslash G}<\overrightarrow{\mathbf{0}}_{\Phi \backslash G}$ по переменным $\left.\overrightarrow{\mathbf{p}}\right|_{\Phi \backslash G}$, а их производные продолжаются до непрерывных функиий в замыкании страта; предельные значения производных являются гладкими в стра$\max \partial_{G_{1}} \wp, G \subset G_{1}$ и аналитическими в области $\left.\operatorname{Re} \Delta \overrightarrow{\mathbf{p}}\right|_{\Phi \backslash G_{1}}<\overrightarrow{\mathbf{0}}_{\Phi \backslash G_{1}}$.

3. В $\partial_{G \wp}$ норма $\partial \overrightarrow{\mathbf{s}}_{\operatorname{Im}} \overrightarrow{\mathbf{p}}(\overrightarrow{\mathbf{p}}, \mu) / \partial \overrightarrow{\mathbf{p}}$ менъше единицъ. Отображение $\overrightarrow{\mathbf{h}}(\overrightarrow{\mathbf{p}}, \mu)$ взаимно одназначно при фиксированном $\mu$.

4. Образ ю при отображении $(\overrightarrow{\mathbf{p}}, \mu) \rightarrow(\overrightarrow{\mathbf{h}}(\overrightarrow{\mathbf{p}}, \mu), \mu)$ содержит $\mathbb{C}^{\Phi} \times \mathcal{M}$. Образы стратов $\partial_{G \wp}-C^{\infty}$-гладкие многообразия вещественной коразмерности $|G|-1$; образы стратов $\partial_{G} \wp^{\mathbb{C}}$ суть комплексные многообразия $\partial_{G} \Im^{\mathbb{C}}$ комплексной коразмерности $|G|-1$. Обратное отображение $\overrightarrow{\mathbf{p}}(\overrightarrow{\mathbf{h}}, \mu)$ непрерывно на $\Im, C^{\infty}$-гладко в каждом страте $\partial_{G} \Im$ и аналитично в области $\left.\operatorname{Re} \Delta \overrightarrow{\mathbf{p}}(\overrightarrow{\mathbf{h}}, \mu)\right|_{\Phi \backslash G}<\overrightarrow{\mathbf{0}}_{\Phi \backslash G}$, его производные продолжаются до непрерывных в замыкании страта $\partial_{G} \Im$, а предельные значения производных являются гладкими в $\partial_{G_{1}} \Im, G \subset G_{1}$ и аналитическими в области Re $\left.\Delta \overrightarrow{\mathbf{p}}(\overrightarrow{\mathbf{h}}, \mu)\right|_{\Phi \backslash G_{1}}<\overrightarrow{\mathbf{0}}_{\Phi \backslash G_{1}}$.

5. Давление в модели, найденное как термодинамический предел статистических сумм (1) и (2) с устойчивым граничным условием $q$, равно

$$
p_{q}(\overrightarrow{\mathbf{h}}, \mu)=p_{\max , \mathbb{R}}(\overrightarrow{\mathbf{h}}, \mu)+i \operatorname{Im} p_{q}(\overrightarrow{\mathbf{h}}, \mu) .
$$

Оно непрерывно в, аналитично в каждом страте $\partial_{G} \Im^{\mathbb{C}}$ и является гладкой функичей в каждом страте $\partial_{G} \Im$, предельные значения производных давления гладкие в каждом из стратов $\partial_{G_{1}} \Im, G_{1} \subset G$, и аналитические в $\partial_{G_{1}} \Im \mathbb{C}, G_{1} \subset G$. Если $\overrightarrow{\mathbf{h}}=\overrightarrow{\mathbf{h}}(\mu)$, то давления определяются функиией $p_{q}(\mu):=p_{q}(\overrightarrow{\mathbf{h}}(\mu), \mu)$. Страты сосуществования фаз $\partial_{G} \mathcal{M}^{\mathbb{C}}:=\left\{\mu \in \mathcal{M}: \overrightarrow{\mathbf{h}}(\mu) \in \partial_{G} \Im^{\mathbb{C}}\right\}$ являются аналитическими множествами.

6. Для статистических сумм (1) справедливы следующие представления:

$$
\Xi_{q}(V, H)=e^{\left(p_{\max , \mathbb{R}}(\overrightarrow{\mathbf{h}}, \mu)+i \operatorname{Im} p_{q}(\overrightarrow{\mathbf{h}}, \mu)\right)|V|} \Delta_{q}(V) \sigma_{q}(V),
$$

где для $s_{q}, \ln \Delta_{q}, \ln \delta_{q}$ сходятся соответствующие кластерные разложения (16). Для устойчивых фаз $\sigma_{q}(V)=1, \Xi_{q}(V, H)=e^{p_{q}|V|} \Delta_{q}(V) u$

$$
\left|\ln \Xi_{q}(V, H)-p_{q}\right| V|| \leqslant C \psi_{0}^{2 \nu}|\partial V|, \quad q \in G(\mu) .
$$

7. Гиббсовские состояния, построенные по граничному условию $q \in G(\mu)$, являются малым возмущением фбазы q (теорема I.5). При пересечение страта фазовой диаграммы в пространстве параметров одна из производных давления по $\overrightarrow{\mathbf{h}}$ разрывна.

8. Справедлив критерий устойчивости фаз (теорема 2).

9. Оченки производных различных величин определяются теоремой 5.

10. В области фазовых переходов первого рода статистические суммы с устойчивым граничным условием не имеют нулей (обобщение теоремы Ли-Янга). 


\section{ПРИЛОЖЕНИЕ}

1. Доказательство теоремы 2. Доказательство теоремы 2 основано на уточнении оценки величины $\sigma_{q}(V)$ в $(24)$ по сравнению с той, которая была использована при доказательстве теоремы I.4. Как и при доказательстве теоремы I.4, мы оцениваем величину $\sigma_{q}(V)$ суммой вида (ниже в этом разделе $b \equiv b_{q}^{e}, V$ - куб)

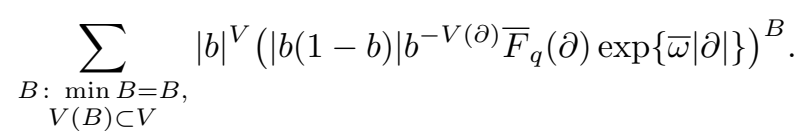

Используя оценки приложения к работе [26] для статистических сумм газа "твердых объемов", имеем, что эта сумма не превосходит $d_{m}, m=\max _{V(\partial) \subset V, U_{0}(\partial, \partial V)=0}|V(\partial)|$. Последовательность $d_{j}, j=1,2, \ldots$, определена рекуррентно:

$$
d_{1}=b^{2}, \quad d_{j}=d_{j-1}^{(j+1) / j}+t_{j}, \quad t_{j}:=b(1-b) e^{-\tau j^{(\nu-1) / \nu}}, \quad e^{-\tau}=C_{4} \psi_{0} .
$$

В разделе I.5 мы использовали оценку $d_{m} \leqslant 1$, легко вытекающую из (67) при достаточно больших $\tau$. Оказывается, последовательность $d_{j}$ в основном правильно описывает поведение статистических суммы с неустойчивым граничным условием в зависимости от внешнего поля, энергетического барьера $\tau$ и величины объема $j$. В частности, с ростом $j$ при $b$, близком к единице, и больших $\tau$ у последовательности $d_{j}$ есть два режима поведения: при малых $j$ в рекуррентном соотношении превалирует первое слагаемое и $d_{j} \approx b^{j+1}$, а для достаточно больших $j$ первое слагаемое становится меньшим второго и тогда $d_{j} \approx e^{-\tau_{1} j^{(\nu-1) / \nu}}$. Для доказательства теоремы 2 достаточно следующей леммы $(h:=-\ln b)$.

Лемма 4. Найдется достаточно большое $\tau_{0}$, зависящее лишь от $\nu$, такое, что при $\tau \geqslant \tau_{0}, b<1, j \geqslant i_{0}=(\tau /(2 h))^{\nu}$ для последовательности (67) справедливо неравенство $d_{j} \leqslant e^{-j^{(\nu-1) / \nu} \tau / 2}$. Кроме того, $d_{j} \leqslant 1$ для всех $j$.

ДокАЗАтельство. Заметим, что $d_{j} \geqslant b^{j+1}$. Следовательно,

$$
\ln d_{j} \leqslant \frac{(j+1)}{j} \ln d_{j-1}+\frac{t_{j}}{d_{j-1}^{(j+1) / j}} \leqslant \cdots \leqslant(j+1)\left(-h+\sum_{k=1}^{j+1} \frac{t_{k}}{k b^{k}}\right) .
$$

Последнюю сумму можно записать как $\sum_{k=1}^{j} e^{-\tau k^{1-1 / \nu}+h k} / k$. Для $j=i_{0}=(\tau /(2 h))^{\nu}$ имеем

$$
\sum_{k=1}^{i_{0}} \frac{e^{-\tau k^{1-1 / \nu}+h k}}{k} \leqslant \sum_{k=1}^{i_{0}} \frac{e^{-(\tau / 2) k^{1-1 / \nu}}}{k} \leqslant e^{-\tau_{1}}
$$

где

$$
2 \tau_{1}=\tau-C(\tau), \quad C(\tau)=\ln \sum_{k=1}^{\infty} \frac{1}{k} e^{-(\tau / 2)\left(k^{1-1 / \nu}-1\right)} \underset{\tau \rightarrow \infty}{\rightarrow} 0 .
$$

Тем самым $\ln d_{i_{0}} \leqslant i_{0}\left(-h+b(1-b) e^{-\tau_{1}}\right) \leqslant-\tau i_{0}^{(\nu-1) / \nu} / 2$. Для того чтобы неравенство леммы выполнялось для $j+1$, если оно верно для $j$, достаточно, как это вытекает из (67), чтобы были выполнены неравенства

$$
e^{-\tau j^{1-1 / \nu}(1+1 / j) / 2}+b(1-b) e^{-\tau(j+1)^{1-1 / \nu}} \leqslant e^{-\tau(j+1)^{1-1 / \nu} / 2},
$$


или

$$
e^{\left(1+1 / j-(1+1 / j)^{1-1 / \nu}\right)\left(-\tau\left(j^{1-1 / \nu}\right) / 2\right.}+b(1-b) e^{-\tau(j+1)^{1-1 / \nu} / 2} \leqslant 1,
$$

или

$$
e^{-\tau /\left(2 \nu j^{1 / \nu}\right)}+b(1-b) e^{-\tau(j+1)^{1-1 / \nu} / 2} \leqslant 1 .
$$

Так как производная левой части последнего неравенства отрицательна при больших $\tau$, лемма 4 доказана.

2. Оценка функций Урселла и их сумм. Приведем стандартную связь между гамильтонианом и потенциалами в классических моделях статистической физики. Пусть $X$ - конечное множество (множество “частиц") и для каждого $Y \subset X$ определено число $H(Y)$ - гамильтониан или энергия взаимодействия частиц $Y$. Многочастичные потенциалы и гамильтониан связаны соотношениями

$$
H(Y)=\sum_{Y_{1} \subset Y} W\left(\left[Y_{1}\right]\right), \quad W([Y])=\sum_{Y_{1} \subset Y}(-1)^{\left|Y \backslash Y_{1}\right|} H\left(Y_{1}\right) .
$$

Пусть $A$ - разбиение $Y(A \in R[Y], X(A)=Y)$. Тривиальное разбиение $Y$, состоящее из $|Y|$ элементов, обозначим $[Y]$. Определим множество разбиений $A$ как те $C \in$ $R[X(A)]$, каждый элемент которых является объединением некоторых групп из $A$, $C \in R A$. Определим условный гамильтониан и энергию взаимодействия групп $A$ при условии $Y_{1}$ как соответственно

$$
\begin{gathered}
H\left(Y \mid Y_{1}\right):=H\left(Y \cup Y_{1}\right)-H\left(Y_{1}\right), \quad Y \cap Y_{1}=\varnothing, \\
H\left(A \mid Y_{1}\right):=H\left(X(A) \mid Y_{1}\right)-\sum_{Y_{2} \in A} H\left(Y_{2} \mid Y_{1}\right), \quad Y_{1} \cap X(A)=\varnothing .
\end{gathered}
$$

Легко видеть, что $H\left(A \mid Y_{1}\right)=\sum_{B \subset A} W\left(B \mid Y_{1}\right)$, где

$$
\begin{aligned}
W\left(B \mid Y_{1}\right) & =\sum_{B_{1} \subset B}(-1)^{\left|B \backslash B_{1}\right|} H\left(B \mid Y_{1}\right)=\sum_{B_{1} \subset B}(-1)^{\left|B \backslash B_{1}\right|} H\left(X\left(B_{1}\right) \cup Y_{1}\right)= \\
& =\sum_{\substack{Y_{2} \subset X(B) \cup Y_{1}: \\
Y_{2} \cap Y_{3} \neq \varnothing \forall Y_{3} \in B}} W\left(\left[Y_{2}\right]\right) .
\end{aligned}
$$

Так как для всех $x \in Y_{2} \in A$ выполнено тождество

$$
W\left(A \mid Y_{1}\right)=W\left(Y_{2} \backslash x, A \backslash Y_{2} \mid Y_{1}\right)+W\left(x, A \backslash Y_{2} \mid Y_{1} \cup Y_{2} \backslash x\right),
$$

получаем, что

$$
W\left(A \mid Y_{1}\right)=\sum_{\substack{Y \subset X(A): \\\left|Y \cap Y_{2}\right|=1 \forall Y_{2} \in A}} W\left([Y] \mid Y^{1}(Y) \cup Y_{1}\right),
$$

где $Y^{1}(Y) \cap Y=\varnothing$ и $Y^{1}(Y)$ определяется последовательным выбором $x \in Y_{2} \in A$ в предпоследнем тождестве. Заметим также, что $H([Y])=H([Y \backslash x])+W(x, Y \backslash x)$, откуда при любом упорядочивании элементов $Y=\left\{y_{1}, \ldots, y_{k}\right\}$ имеем представление

$$
H([Y])=\sum_{y \in Y \backslash y_{k}} W(y, Y(y)),
$$


где $Y\left(y_{i}\right)=\left\{y_{i+1}, \ldots, y_{k}\right\}$. Из последнего представления, очевидно, вытекает неравенство

$$
\operatorname{Re} H([Y]) \geqslant-\sum_{y \in Y \backslash y_{0}} B(y)=:-B\left(Y \backslash y_{0}\right),
$$

где $B(y):=\max _{X, Y: Y \subset X \backslash y}\{0, \operatorname{Re}-W(y, Y)\}$.

Напомним, что функции Урселла определены следующим образом:

$$
\varphi\left(A \mid Y_{1}\right):=\sum_{C \in R A}(-1)^{|C|}(|C|-1) ! \prod_{Y \in C} e^{-\beta H\left([Y]_{A} \mid Y_{1}\right)},
$$

где $[Y]_{A}$ - разбиение $Y$ на группы из $A$. Воспользуемся $\beta$-представлением функций Урселла, полученным в [27] для гамильтонианов с двухчастичным потенциалом. Дифференцируя по $\beta$ функцию Урселла и, по существу, повторяя вывод соотношения (6) в [27], находим

$$
\varphi\left(A \mid Y_{1}\right)=-\left.\int_{0}^{\beta} d \beta_{1} \sum_{C \subset A} W\left(C \mid Y_{1}\right) e^{-\beta_{1} H\left(C \mid Y_{1}\right)} \varphi\left(\widehat{C} A \mid Y_{1}\right)\right|_{\beta=\beta_{1}},
$$

где $\widehat{C} A$ - такое разбиение из $R A$, в котором по сравнению с $A$ объединены в одно целое группы из $C$. Итерации предыдущего тождества дают

$$
\begin{aligned}
& \varphi\left(A \mid Y_{1}\right)=\sum_{l=1}^{|A|-1}(-1)^{l} \int_{0 \leqslant \beta_{1} \leqslant \cdots \leqslant \beta_{l} \leqslant \beta}(d \beta)^{l} \sum_{C_{1} \subset A,} \prod_{i=1}^{l} W\left(C_{i}\right) e^{-\beta_{i} H\left(C_{i} \mid Y_{1}\right)} . \\
& C_{2} \subset \widehat{C}_{1} A \text {, } \\
& \dddot{C}^{i}=X(A)
\end{aligned}
$$

Как и в [27], можно убедиться, что

$$
\sum_{i=1}^{k} H\left(C_{i} \mid Y_{1}\right)=\sum_{Y \in \widehat{C}^{k} A} H\left([Y]_{A} \mid Y_{1}\right)
$$

Перейдя к переменным $t_{k}=\beta_{k}-\beta_{k+1} \geqslant 0$, показатель экспоненты в (72) выразим через $t_{k}$, воспользуемся также предыдущим тождеством и неравенством (71), тогда

$$
\operatorname{Re} \sum_{i=1}^{l} \beta_{i} H\left(C_{i} \mid Y_{1}\right)=\operatorname{Re} \sum_{k=1}^{l} t_{k} \sum_{Y \in \widehat{C}^{k} A} H\left([Y]_{A} \mid Y_{1}\right) \geqslant-\beta B\left(X \backslash x_{0}\right) .
$$

Введем функции

$$
\bar{W}(Y):=\max _{Y_{1} \subset X \backslash y}\left|W\left([Y] \mid Y_{1}\right)\right|, \quad \bar{W}^{(k)}(Y):=\max _{Y_{1} \subset X \backslash y}\left|W^{(k)}\left([Y] \mid Y_{1}\right)\right| .
$$

Отметим, что из последнего определения и формулы (69) получаем

$$
\left|W^{(k)}(A)\right| \leqslant \sum_{Y \cap A \neq \varnothing} \bar{W}^{(k)}(Y)=: \bar{W}^{(k)}(A), \quad k \geqslant 0,
$$

где неравенство $k \geqslant 0$ является покомпонентным; $Y \stackrel{*}{\cap} A \neq \varnothing$ означает, что $\left|Y \cap Y_{2}\right|=1$ для всех $Y_{2} \in A$. Обозначим $\left\{\gamma_{x_{0}}(X)\right\}$ множество “обобщенных деревьев" с корнем 
$x_{0}$, множеством вершин $X$ и множеством “обобщенных линий” $\pi(\gamma)$, где каждой "обобщенной линии” сопоставлен потенциал $\bar{W}(Y)$. Теперь нетрудно получить необходимые оценки.

Лемма 5. Имеют место следующие утверждения.

1. Справедливы оченки

$$
|\varphi(X)| \leqslant e^{B\left(X \backslash x_{0}\right)} \sum_{\gamma_{x_{0}}(X)} \prod_{Y \in \pi(\gamma)} \bar{W}(Y)
$$

и соответствующие оценки производных, в которых формальная производная В равна

$$
B^{(k)}(y):=\max _{Y \subset X \backslash y}\left|W^{(k)}(y, Y)\right|, \quad k \neq 0 .
$$

2. Мажорантными оценками сумм относительно $z$, т.е. оценками для

$$
\bar{\rho}:=\sum_{X \ni x_{0}}\left|z^{X} \varphi([X])\right| e^{-U^{0}(X)}, \quad \bar{\rho}^{(k)}:=\sum_{X \ni x_{0}}\left|\left(z^{X} \varphi(X)\right)^{(k)}\right| e^{-U^{0}(X)},
$$

в которых $U_{0}(X):=\sum_{x, y \in X} U_{0}(x, y), U_{0}(x, y)=0, \infty$, являются решения уравнений

$$
\bar{\omega}(x)=|z(x)| \exp \left\{\sum_{X} \bar{W}(x \cup X)\left(\bar{\omega} e^{B}\right)^{X} e^{-U^{0}(x \cup X)}\right\} .
$$

Кроме того, если найдутся величины $\bar{\omega}_{0}^{(k)}(x)$ такие, что

$$
\left\{|z(x)| \exp \left\{\sum_{X} \bar{W}(x \cup X)\left(\bar{\omega}_{0} e^{B}\right)^{X} e^{-U^{0}(x \cup X)}\right\}\right\}^{(k)} \leqslant \bar{\omega}_{0}^{(k)}(x),
$$

то $\bar{\rho}^{(k)} \leqslant \bar{\omega}_{0}^{(k)}(x)$, и соответствующие рядъ (76) сходятся.

Доказательство первого утверждения леммы вытекает из представления (72), а также формул (69), (71)-(75). Формула (77) задает производящую функцию “деревьев" и соответствующих производных, откуда получаем второе утверждение леммы.

3. Оценки производных произвольного порядка. Для анализа фазовой диаграммы необходимы оценки не только первых, но и вторых производных термодинамических функций. Сначала рассмотрим двухкомпонентную модель с контурным функционалом $\mathcal{F}_{q}(\partial)$ и нулевым внешним полем (см. раздел I.4) и обобщим п. 3 теоремы I.3 (индекс $q$ в лемме 6 фиксирован).

Лемма 6. Если найдутся положителъные величины $\bar{\omega}_{q}^{(k)}, 0 \leqslant k<k_{0},\left(k, k_{0}-\right.$ мультииндексъ, неравенства понимаются как покомпонентные) такие, что

$$
\sum_{\partial{ }_{\cap}^{*} 0 \neq \varnothing}\left[\left|\mathcal{F}_{q}(\partial)\right| e^{\bar{\omega}_{q}|\partial|}\right]^{(k)} \leqslant \bar{\omega}_{q}^{(k)},
$$

где $[\cdot]^{(k)}-k$-я формальная производная в левой части (78), то справедливь следующ,е мажорантные оценки относительно $\mathcal{F}_{q}(\partial)$ и производных $\mathcal{F}_{q}(\partial)$ :

$$
\begin{gathered}
\left|s_{q}^{(k)}\right| \leqslant \bar{\omega}_{q}^{(k)}, \quad\left|\Delta_{q}^{(k)}(V)\right| \leqslant\left(e^{\bar{\omega}_{q}|\partial V|}\right)^{(k)}, \quad\left|\delta_{q}^{(k)}(\partial \cup B)\right| \leqslant\left(e^{\bar{\omega}_{q}\|B\|}\right)^{(k)}, \\
\left|\ln ^{(k)} \Delta_{q}(V)\right| \leqslant \bar{\omega}_{q}^{(k)}|\partial V|, \quad\left|\ln ^{(k)} \delta_{q}(\partial \cup B)\right| \leqslant \bar{\omega}_{q}^{(k)}\|B\| .
\end{gathered}
$$


Теперь рассмотрим контурные уравнения (17) с уравнениями на производные от величин $F_{q}(\partial), \sigma_{q}(V), \mathcal{F}_{q}(\partial)$, полученных дифференцированием (17) по параметрам модели. Предположим, что найдутся величины $\bar{\omega}_{q}^{(k)}, a_{q}^{(k)}$ такие, что справедливы неравенства

$$
\begin{gathered}
\left|\Delta_{q}^{(k)}(V)\right| \leqslant\left(e^{\bar{\omega}_{q}|\partial V|}\right)^{(k)}, \\
\left|\delta_{q}^{(k)}(\partial \cup B)\right| \leqslant\left(e^{\bar{\omega}_{q}\|B\|}\right)^{(k)}, \\
\left|\sigma_{q}^{(k)}(V)\right| \leqslant\left(b^{V} e^{a_{q}|V|}\right)^{(k)}\left(b e^{a_{q}}\right)^{-V}=: \bar{\sigma}_{q}^{(k)}(V), \\
\bar{\sigma}_{q}^{(0)}(V):=b^{m_{1}(\partial V)}, \quad b=\left|b_{q}^{e}\right| .
\end{gathered}
$$

Оценкой функционалов $F_{q}^{(k)}(\partial), \mathcal{F}_{q}^{(k)}(\partial)$ при $k \neq 0$ являются функционалы

$$
\begin{aligned}
& \bar{F}_{q}^{(k)}(\partial):=\sum_{\vec{\partial}^{\mathrm{ex}}=\partial_{q}}\left|\varphi_{\overrightarrow{\boldsymbol{\partial}}} e^{-p V_{\vec{\partial}}+\bar{\omega} \vec{\partial}} \bar{\sigma}(\vec{\partial})\right|^{(k)} \\
& \overline{\mathcal{F}}_{q}^{(k)}(\partial):=\left(\bar{F}_{q}(\partial) \bar{\sigma}_{q}(V(\partial))\right)^{(k)}
\end{aligned}
$$

где

$$
\bar{\omega} \overrightarrow{\boldsymbol{\partial}}:=\sum_{i \in \bar{l}(\overrightarrow{\boldsymbol{\partial}})} \bar{\omega}_{k_{i}}\left|\partial V_{i}(\overrightarrow{\boldsymbol{\partial}})\right|, \quad \bar{\sigma}(\overrightarrow{\boldsymbol{\partial}})=\prod_{i \in l(\overrightarrow{\boldsymbol{\partial}})} \bar{\sigma}_{k_{i}(\overrightarrow{\boldsymbol{\partial}})}\left(V_{i}(\overrightarrow{\boldsymbol{\partial}})\right) .
$$

Правая часть (81) выписана из уравнений (17) после использования в них (80). Условие согласованности оценок с контурным уравнением (17) имеет вид

$$
\begin{gathered}
\sum_{\partial \stackrel{*}{\cap} 0 \neq \varnothing} \overline{\mathcal{F}}_{q}^{(k)}(\partial) \leqslant \bar{\omega}_{q}^{(k)}, \\
\sum_{B: V(B) \subset V}\left|\bar{F}_{q}^{B} \varphi_{\partial V \cup B}^{q} e^{\bar{\omega}_{q}\|B\|}\right|^{(k)} \leqslant \bar{\sigma}_{q}^{(k)}(V(\partial)),
\end{gathered}
$$

так как при выполнении (82), как это следует из леммы 5, верны первые два неравенства (80). Как и в доказательстве теоремы I.4, можно получить, что оценка левой части (83) воспроизводится ее правой частью в области $\bar{S}_{1}\left(a_{q}\right)$ (см. $(20)$ ). Действительно, каждое слагаемое в сумме в правой части (83) можно представить в виде произведения сомножителей, в каждом из которых есть лишь один минимальный контур из $B$. После взятия $k$-й производной мы фиксируем те сомножители, которые дифференцируются, по оставшимся сомножителям проводится суммирование и используются те же оценки, что и перед леммой 4; сумма этих величин без фиксированных сомножителей не превосходит единицы, если $b_{q}^{e} \in \bar{S}_{1}\left(a_{q}\right)$. Затем суммируем по тем $k_{1}$ сомножителям, которые дифференцировались. Это дает величину, не превосходящую $|V|^{k_{1}}$, умноженную на соответствующие производные. Нетрудно видеть, что оценка левой части (83) воспроизводится правой частью (83), где $a_{q} \leqslant \tilde{a}$ (см. (49)) и более точно определено формулой (I.56). Увеличив еще более $a_{q}$, можно взять $a_{q}=\bar{\omega}_{1, q} e^{\bar{\omega}_{V, q}}$, где

$$
\bar{\omega}_{1, q}:=\sum_{\partial: 0 \in V\left(\partial^{q}\right)} 2\left|V\left(\partial^{q}\right)\right| \bar{F}_{q}(\partial) e^{\bar{\omega}_{q}\left|\partial_{q}\right|}, \quad \bar{\omega}_{V, q}:=\sum_{\partial^{q}: 0 \in V\left(\partial^{q}\right)}\left|b_{q}^{e}\right|^{V\left(\partial^{q}\right)} \bar{F}_{q}(\partial) e^{\bar{\omega}_{q}|\partial|} .
$$

Итак мы получили следующее утверждение. 
ТЕорема 5 (оценки производных). Если найдутся величины $\bar{\omega}_{q}^{(k)} \geqslant 0,0 \leqslant k<$ $k_{0}, k_{0} \leqslant \infty$, такие, что выполнено (82), (83), то величины $s_{q}, F_{q}(\partial), \ln \Delta_{q}, \ln \delta_{q}$, $\sigma_{q}$ являются $k_{0}-1$ раз непрерывно дифферениируемыми функииями в замыкании каждого страта фазовой диаграммы. Для этих величин выполнены неравенства (79), (80) и неравенство $\left|\mathcal{F}_{q}^{(k)(\partial)}\right| \leqslant \overline{\mathcal{F}}_{q}^{(k)}(\partial)$.

Благодарности. Автор выражает искреннюю благодарность Г. П. Мещеряковой за чтение рукописи и ряд ценных замечаний по ней.

\section{Список литературы}

[1] С. А. Пирогов, Я. Г. Синай, ТМФ, 25:3 (1975), 358-369; $26: 1$ (1976), 61-76.

[2] J.Z. Imbrie, Commun. Math. Phys., 82:2 (1981), 261-304; 82:3, 305-343.

[3] M. Zahradnik, Commun. Math. Phys., 93:4 (1984), 559-581.

[4] R. Kotecký, D. Preiss, Rend. Circ. Mat. Palermo (2), 1984, № 3(suppl.), 161-164.

[5] А. Г. Басуев, ТMФ, 58:2 (1984), 261-278.

[6] J. Bricmont, K. Kuroda, J. L. Lebowitz, Commun. Math. Phys., 101:4 (1985), 501-538.

[7] J. Fröhlich, A. Bovier, U. Glaus, "Mathematical aspects of the physics of disordered systems", Critical Phenomena, Random Systems and Gauge Theories (Les Houches, France, 1984), North-Holland, Amsterdam, 1986, 725-893.

[8] J. Slawny, "Low-temperature properties of classical lattice systems: phase transitions and phase diagrams", Phase Transitions and Critical Phenomena, vol. 11, eds. C. Domb, J. L. Lebowitz, Academic Press, London-New York, 1987, 127-205.

[9] А. Г. Басуев, ТМФ, 64:1 (1985), 103-129; 72:2 (1987), 255-268.

[10] R. L. Dobrushin, M. Zagradnik, "Phase diagramm for continuous spin models: An extension Pirogov-Sinai theory", Mathematical Problems of Statistical Mechanics and Dynamics, Math. Appl. (Soviet Ser.), 6, ed. R. L. Dobrushin, Reidel, Dordrecht, 1986, 1-123.

[11] F. Koukiou, D. Petritis, M. Zahradnik, Commun. Math. Phys., 118:3 (1988), 365-383.

[12] Yong Moon Park, Commun. Math. Phys., 114:2 (1988), 219-241.

[13] J. Bricmont, J. Slawny, J. Statist. Phys., 54:1-2 (1989), 89-161.

[14] J. Fröhlich, L. Rey-Bellet, D. Ueltschi, Commun. Math. Phys., 224:1 (2001), 33-63.

[15] M. Zahradnik, J. Statist. Phys., 47:5-6 (1987), 725-755.

[16] A. C. D. van Enter, R. Fernández, A. D. Sokal, J. Statist. Phys., 72:5-6 (1993), 879-1167.

[17] R. H. Schonmann, N. Yoshida, Commun. Math. Phys., 189:2 (1997), 299-309.

[18] M. Biskup, C. Borgs, J. T. Chayes et al., Commun. Math. Phys., 251:1 (2004), 79-131.

[19] S. N. Isakov, Commun. Math. Phys., 95:4 (1984), 427-443.

[20] С. Н. Исаков, ТМФ, 71:3 (1987), 426-440.

[21] S. Friedli, Ch.-E. Pfister, Commun. Math. Phys., 245:1 (2004), 69-103.

[22] M.E. Fisher, Arch. Rational Mech. Anal., 17:5 (1964), 377-410.

[23] Д. Рюэль, Статистическая механика. Строгие результать, Мир, М., 1971.

[24] R. B. Griffiths, "Rigorous results and theorems", Phase Transition and Critical Phenomena. Vol. 1. Exact Results, eds. C. Domb, M. S. Green, Academic Press, London-New York, 1972, 7-109.

[25] C. N. Yang, T. D. Lee, Phys. Rev. (2), 87:3 (1952), 404-409; 410-419.

[26] А. Г. Басуев, ТMФ, 58:1 (1984), 121-136.

[27] А. Г. Басуев, ТМФ, 39:1 (1979), 94-105.

Поступила в редакцию 29.09.2006, после доработки 20.03.2007 